ARTICLE

\title{
Xyloglucan processing machinery in Xanthomonas pathogens and its role in the transcriptional activation of virulence factors
}

Plinio S. Vieira (1) 1,9, Isabela M. Bonfim (10 1,2,9, Evandro A. Araujo1,3, Ricardo R. Melo', Augusto R. Lima', Melissa R. Fessel ${ }^{4}$, Douglas A. A. Paixão', Gabriela F. Persinoti (1) 1, Silvana A. Roccoㄴ, Tatiani B. Lima1, Renan A. S. Pirolla', Mariana A. B. Morais (1) 1, Jessica B. L. Correa', Leticia M. Zanphorlin', Jose A. Diogo 1,2, Evandro A. Lima', Adriana Grandis ${ }^{6}$, Marcos S. Buckeridge ${ }^{6}$, Fabio C. Gozzo ${ }^{7}$, Celso E. Benedetti ${ }^{5}$, Igor Polikarpov (1) ${ }^{8}$, Priscila O. Giuseppe (i) ${ }^{1 凶} \&$ Mario T. Murakami (i) ${ }^{1 凶}$

Xyloglucans are highly substituted and recalcitrant polysaccharides found in the primary cell walls of vascular plants, acting as a barrier against pathogens. Here, we reveal that the diverse and economically relevant Xanthomonas bacteria are endowed with a xyloglucan depolymerization machinery that is linked to pathogenesis. Using the citrus canker pathogen as a model organism, we show that this system encompasses distinctive glycoside hydrolases, a modular xyloglucan acetylesterase and specific membrane transporters, demonstrating that plant-associated bacteria employ distinct molecular strategies from commensal gut bacteria to cope with xyloglucans. Notably, the sugars released by this system elicit the expression of several key virulence factors, including the type III secretion system, a membrane-embedded apparatus to deliver effector proteins into the host cells. Together, these findings shed light on the molecular mechanisms underpinning the intricate enzymatic machinery of Xanthomonas to depolymerize xyloglucans and uncover a role for this system in signaling pathways driving pathogenesis.

\footnotetext{
${ }^{1}$ Brazilian Biorenewables National Laboratory (LNBR), Brazilian Center for Research in Energy and Materials (CNPEM), Campinas, São Paulo, Brazil. ${ }^{2}$ Graduate Program in Functional and Molecular Biology, Institute of Biology, University of Campinas, Campinas, São Paulo, Brazil. ${ }^{3}$ Brazilian Synchrotron Light Laboratory (LNLS), Brazilian Center for Research in Energy and Materials (CNPEM), Campinas, São Paulo, Brazil. ${ }^{4}$ Butantan Institute, Butantan Foundation, São Paulo, São Paulo, Brazil. ${ }^{5}$ Brazilian Biosciences National Laboratory (LNBio), Brazilian Center for Research in Energy and Materials (CNPEM), Campinas, São Paulo, Brazil. ${ }^{6}$ Department of Botany, Institute of Biosciences, University of São Paulo, São Paulo, Brazil. ${ }^{7}$ Institute of Chemistry, University of Campinas, Campinas, São Paulo, Brazil. ${ }^{8}$ São Carlos Institute of Physics, University of São Paulo, São Carlos, São Paulo, Brazil. ${ }^{9}$ These authors contributed equally: Plinio S. Vieira, Isabela M. Bonfim. ${ }^{凶}$ email: priscila.giuseppe@Inbr.cnpem.br; mario.murakami@lnbr.cnpem.br
} 
X yloglucans (XyGs) comprise a class of highly complex polysaccharides present in the primary cell wall of vascular plants from clubmosses to angiosperms, including all agricultural cultivars ${ }^{1}$. These recalcitrant polysaccharides form an intricate network with cellulose, which is critical for cell wall function and structure, and serves as a physical barrier against pathogen invasion and colonization ${ }^{2}$.

XyGs are structurally and chemically diverse, consisting of a $\beta$ 1,4-linked glucan backbone decorated with $\alpha$-1,6-xylosyl residues, which might have additional decorations such as D-galactose, Lfucose, and L-arabinose, depending on the source at the tissue level in plants ${ }^{3}$. These polysaccharides can also be acetylated and this modification is known to affect their physicochemical properties and interaction with other cell-wall components ${ }^{1,3,4}$.

To cope with XyGs, many microorganisms, such as saprophytes $^{5}$ and commensal bacteria from the human gut ${ }^{6}$, harbor enzymatic toolboxes encoded by a set of physically linked genes known as XyG utilization loci (XyGUL). Plant pathogens from the Xanthomonas genus also encompass a gene cluster predicted to degrade XyGs (Fig. 1). These pathogens exhibit a high tissue and host specificity, colonizing mesophylls or xylem vessels of over 400 distinct monocotyledons and dicotyledons, including many economically important plants such as citrus, cotton, and corn ${ }^{7,8}$. Regardless of the lifestyle and ecological niche specialization, most Xanthomonas species harbors this predicted XyGUL in their genomes, indicating the relevance of this system for these bacteria. However, the molecular mechanisms underpinning $\mathrm{XyG}$ depolymerization and potential biological roles in Xanthomonas and other phytopathogens remain so far elusive.

Therefore, here we used the causal agent of citrus canker Xanthomonas citri pv. citri (X. citri) as a model organism ${ }^{9}$ to investigate the molecular basis of XyG breakdown and its potential involvement in pathogenesis and host-pathogen interactions. Our results show that Xanthomonas XyGUL encodes a highly elaborate enzymatic cascade including distinct activities (acetylesterase, $\alpha$-L-fucosidase, $\beta$-galactosidase, $\alpha$-xylosidase, and xyloglucanase), catalytic mechanisms (inverting and retaining), modes of action (endo and exo), and 3D architectures (multimodular and quaternary arrangements). This machinery notably differs from other known XyGULs, expanding the current knowledge about microbial molecular strategies associated with the depolymerization and utilization of recalcitrant plant polysaccharides. Furthermore, we reveal a link between this enzymatic system and bacterial virulence through a stimulatory effect of its products on the expression of several key virulence factors, including the type III secretion system (T3SS), a needle-like apparatus that inject effector proteins into the plant cell to modulate host responses in favor of bacterial colonization ${ }^{10}$.

\section{Results}

The XyGUL gene architecture in Xanthomonas diverges from Bacteroidetes. Genomic analysis revealed that most Xanthomonas species conserve a predicted XyGUL consisting of two outer membrane TonB-dependent transporters (TBDTs), four glycoside hydrolases belonging to the families GH74, GH31, GH35, GH95 and one esterase with no significant similarity with any carbohydrate esterase family (Fig. 1a, and Supplementary Tables 1 and 2). Adjacent to this cluster, there are common components for xylose metabolism (D-xylulokinase and xylose isomerase) and an inner membrane MFS sugar transporter (Supplementary Figs. 1 and 2). A search in the Polysaccharide Utilization Loci database ${ }^{11}$ did not result in any similar organization in Bacteroidetes, except for the clustering of three or two XyG-related genes in some species. In characterized Bacteroides
XyGULs, the clustering of GH31 and GH95 genes has been observed, but in association with other carbohydrate-active enzymes (CAZymes) ${ }^{6,12}$. The synteny of GH31, GH35, and GH95 genes was reported in the XyGUL from the saprophyte Celvibrio japonicus ${ }^{5}$, but not physically linked to endoxyloglucanases or esterases genes.

The XyGUL is highly conserved across the Xanthomonas genus regardless of the broad range of hosts (monocotyledons and dicotyledons) and tissue specificity (mesophyll or xylem vessels) (Fig. 1b). Few of them have lost the predicted GH74 xyloglucanase, but endoglucanases encoded outside the XyGUL, such as GH5, GH9, and GH12 members, may compensate for its absence (Supplementary Fig. 2). The only exceptions that lack most of XyGUL genes are Xanthomonas species colonizing gramineous monocotyledons such as $X$. oryzae (rice), $X$. translucens (wheat), and $X$. albilineans (sugarcane) (Fig. 1b). This apparent loss of XyG-degrading capacity correlates with the typically lower contents of $\mathrm{XyG}$ in the cell walls of these plants ${ }^{13-16}$. Furthermore, many Xanthomonas species carrying the predicted XyGUL are promoters of several diseases in highly relevant agricultural crops such as corn (X. vasicola pv. vasculorum causing bacterial leaf streak), tomato (X. perforans causing bacterial spot), banana ( $X$. campestris pv. musacearum causing enset wilt), citrus (X. citri pv. citri causing citrus canker), and cabbage $(X \text {. campestris pv. campestris causing black rot })^{17}$ (Fig. 1b, and Supplementary Table 3). These observations indicate that this system might play important roles in supporting a successful infection, which led us to investigate in depth the molecular mechanisms governing XyG processing by Xanthomonas and its potential biological functions.

XyGUL endo-enzyme exploits arginine-carbohydrate interactions. The first enzymatic unit of the Xanthomonas XyGUL is encoded by XAC1770 (named here as XacXeg74) and belongs to the GH74 family, which is known to have high specificity for $\mathrm{XyGs}^{18,19}$. According to kinetic characterization and cleavage pattern analysis, XacXeg74 is an endo-dissociative enzyme generating a broad distribution of xyloglucan oligosaccharides (XyGOs), but preferentially Glc4- and Glc3-based products (Fig. 2a, and Supplementary Fig. 3c). These results indicate that XacXeg74 would accept both X ( $\alpha-\mathrm{D}-\mathrm{Xyl} p-(1,6)-\beta-\mathrm{D}-\mathrm{Gl} c p-(1-)$, and $G(-4)-\beta-D-G l c p-(1-)$ motifs at the -1 subsite, correlating with the presence of a glycine residue (G465) that confers such capacity to group I members of the GH74 family ${ }^{19}$ (Fig. 2b).

To get further insights into $\mathrm{XyG}$ recognition by the Xanthomonas GH74 enzyme, the crystal structure of $X$. campestris pv. campestris GH74 enzyme (XccXeg74, sharing $84 \%$ of sequence identity with XacXeg74) was determined in complex with the disaccharide XG spanning the subsites +1 and +2 (Supplementary Fig. 3a, b, and Supplementary Tables 4 and 5). Structural comparisons with the closest structurally characterized xyloglucanases from Caldicellulosiruptor lactoaceticus (ClGH74A ${ }^{19}$, PDB ID 6P2M and SeqID 39.8\%) and Niastella koreensis (NkGH74 ${ }^{19}$, PDB ID 6P2L, and SeqID 34.2\%) revealed remarkable differences in the molecular basis for substrate recognition. $\mathrm{ClGH} 74 \mathrm{~A}$ and $N k \mathrm{GH} 74$ rely on several $\mathrm{CH}-\pi$ interactions for substrate anchoring, including at least four aromatic residues at the subsites $-3,+1,+3$ and +5 , and possibly two additional aromatic residues forming the subsites -4 (W126) and -5 (W599) in ClGH74A (Fig. 2b). However, except for the -3 (Y128) and the +1 (W396) aromatic-based subsites, the other aromatic residues are absent in Xanthomonas GH74 enzymes, in particular at the +3 and +5 subsites known to be critical for the endo-processive mode of action ${ }^{19}$ (Fig. 2b). The lack of aromatic residues in the subsites +3 and +5 of XccXeg74 


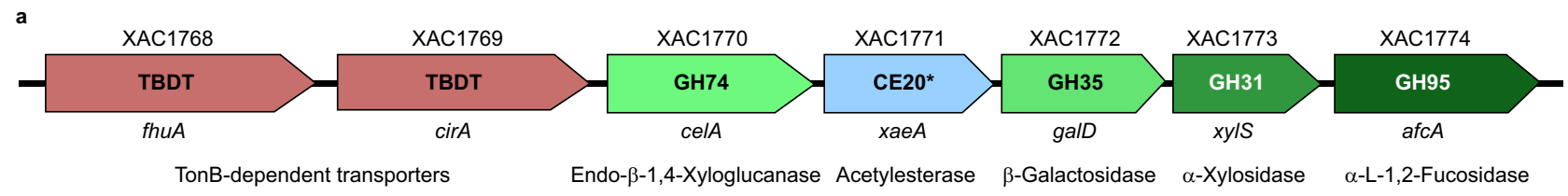

b

$\begin{array}{cl}\text { Host classes } & \text { Local of infection } \\ \text { Dicotyledons } & \text { V Vascular } \\ & \text { M Mesophyllic } \\ \text { Monocotyledons } & \text { U Unknown }\end{array}$

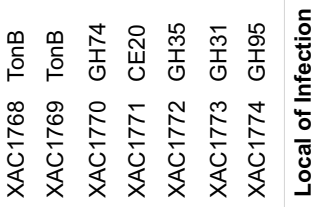

Types of monocotyledons

$\triangle$ Gramineous

$\triangle$ Non-gramineous

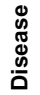

홍
Black rot

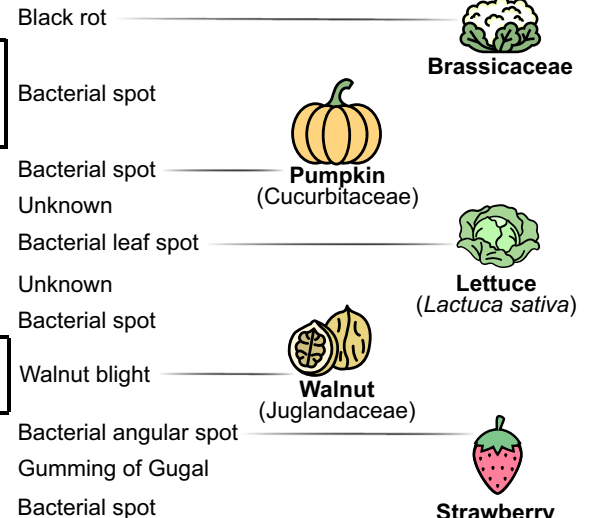

(Fragaria)

Bacterial spot

Bacterial spot

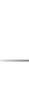
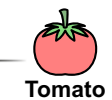

(S. lycopersicum)

Bacterial blight

Bacterial blight

$\int$

Citrus canke

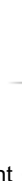

Bacterial blight

Bacterial blight

Bacterial pustule

Bacterial leaf streak

Bacterial leaf streak

Bacterial leaf streak $\quad \begin{gathered}\text { Corn } \triangle \\ \text { (Zea mays) }\end{gathered}$

Enset wilt
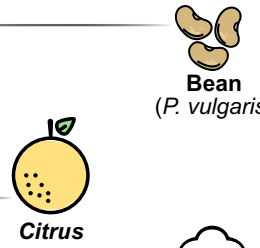

(P. vulgaris

Citrus

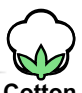

(Gossypium sp.)

X. citri pv. glycines str. 12-2

X. vasicola NCPPB 1060

$X$. vasicola pv. vasculorum $\mathrm{Xv} 1601$

X. vasicola NCPPB 902

X. vasicola pv. arecae NCPPB 2649

X. oryzae BAI23

X. oryzae pv. oryzae PXO99A

$X$. oryzae pv. oryzicola BLS256

-- X. translucens pv. translucens DSM 18974

$X$. translucens pv. undulosa P3

X. hyacinthi CFBP 1156

X. sacchari R1

$X$. albilineans $\mathrm{Xa-FJ1}$

X. albilineans GPE PC73
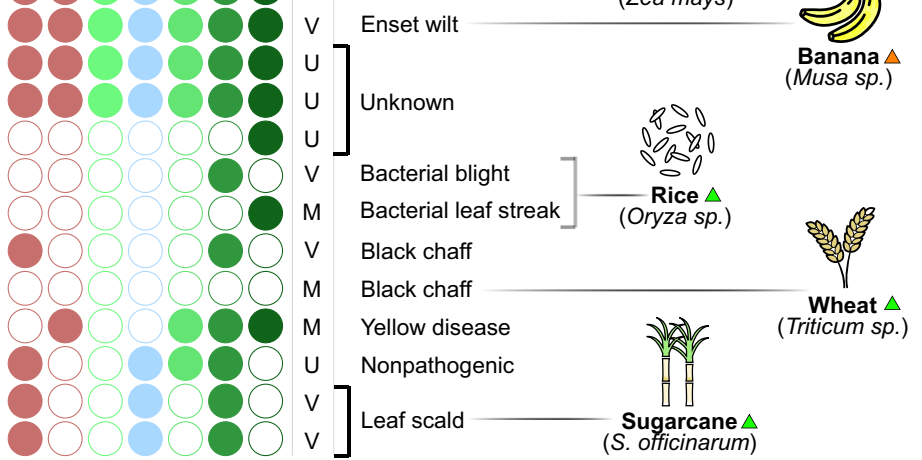

Fig. 1 The XyGUL conservation in Xanthomonas spp. a XyGUL predicted in X. citri pv. citri 306 genome showing TonB-dependent transporters (TBDT in pink), glycoside hydrolases (GHs in shades of green) and a carbohydrate esterase (CE in blue, ${ }^{*}$ revealed in this work). b Dendrogram of Xanthomonas species based on phylogenetic analysis (details in Supplementary Fig. 2) showing the presence (filled circles) or absence (open circles) of XyGUL genes and information about disease and tissue/host specificity. $V=$ vascular, $M=$ mesophyllic and $U=$ unknown. Some species infect dicotyledons (pink box), whereas others colonize monocotyledons (blue box). Monocotyledons are subdivided in gramineous (green triangle) or non-gramineous (orange triangle). 

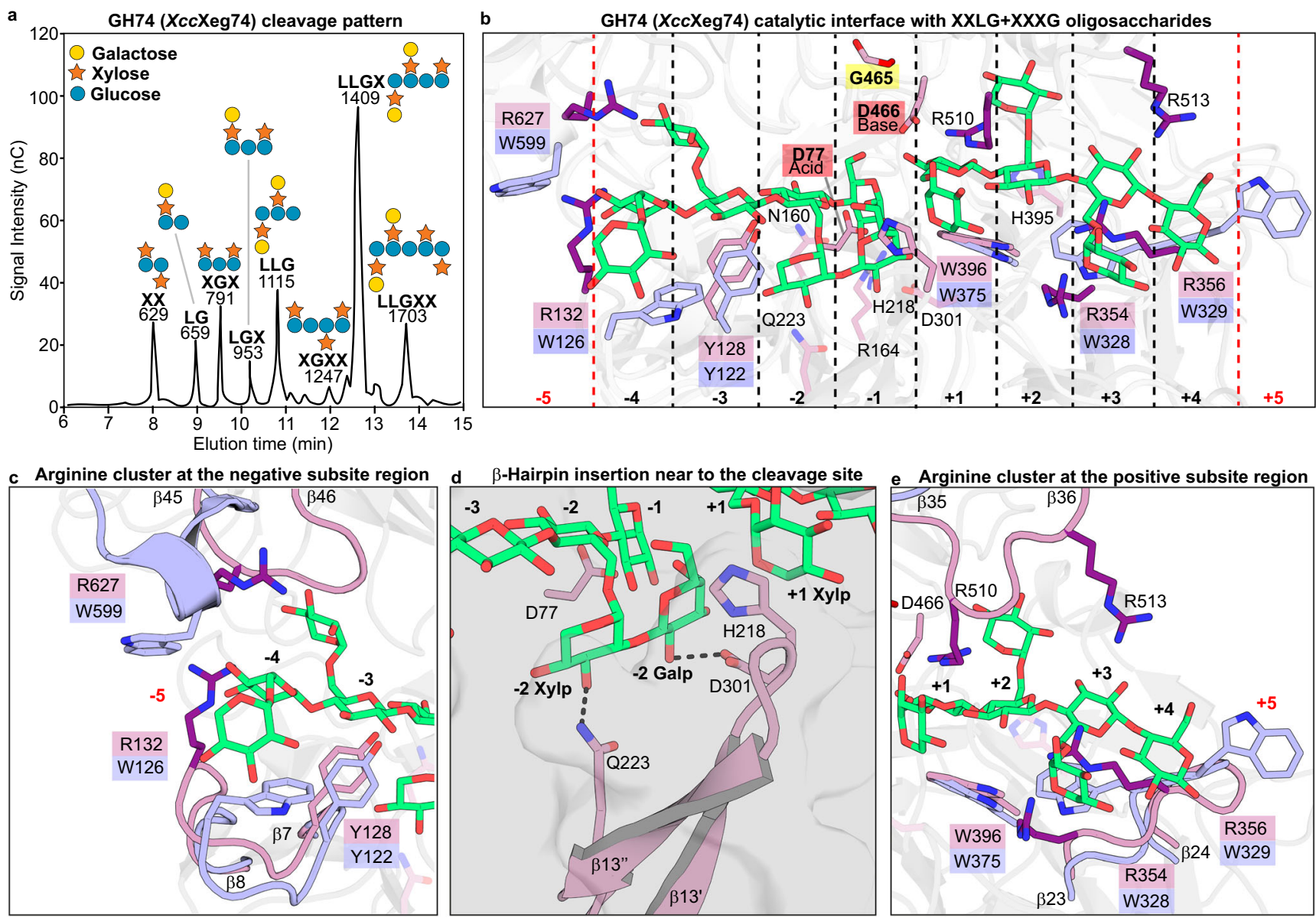

Fig. 2 The structural determinants for substrate recognition in Xanthomonas $\mathbf{G H 7 4}$ endo- $\boldsymbol{\beta}-\mathbf{1 , 4} \mathbf{4}$-xyloglucanases. a HPAEC-PAD analysis of products released from Copaifera langsdorfiii XyG by XccXeg74. Numbers above the peaks represent $m / z$ values of each product assessed by mass spectrometry (details in Supplementary Fig. 3c). Letters indicate the type of substitutions appended to the glucose backbone: $G=$ non-substituted glucose, $X=$ glucose substituted with a xylose at $\mathrm{C}-6$, and $\mathrm{L}=\mathrm{X}$ with a galactose appended at xylose $\mathrm{C}-2$. Symbols represent glucose (blue circle), xylose (orange star), and galactose (yellow circle). b XccXeg74 crystallographic structure (carbon atoms in dark and light purple) superimposed onto the C. lactoaceticus GH74 enzyme (carbon atoms in light blue; PDB ID 6P2 $\mathrm{M}^{19}$ ), and N. koreensis GH74 in complex with XyGOs (only XyGOs shown; carbon atoms in green; PDB ID $6 \mathrm{P} 2 \mathrm{~L}^{19}$ ) highlighting the substrate-binding subsites ( -4 to +4 in XccXeg74 and -5 to +5 in $\mathrm{ClGH74A}$ ). Residues involved in substrate recognition are shown as sticks, with the labels of catalytic residues from XccXeg74 highlighted with red boxes and that of the glycine featuring acceptance to $X$ and $G$ motifs indicated with a yellow box. Note the arginine residues (carbon atoms in dark purple) populating the several substrate-binding subsites in XccXeg74. c, d, e Amplified view of specific zones of the GH74 catalytic interface highlighting structural adaptations found in XccXeg74 that are divergent from CIGH74A.

is, therefore, consistent with its endo-dissociative mode of action on XyG.

XccXeg74 contains three conserved motifs that could compensate for the lack of aromatic platforms in specific subsites: two arginine residues (R132 and R627) at the -4 subsite, a $\beta$-hairpin (residues A211-G226) inserted in the $\eta 2-\beta 14$ loop at the $\mathrm{N}$ terminal lobe, and an arginine cluster at the positive subsite region (Fig. 2b-e, and Supplementary Fig. 4). The $\beta$-hairpin interacts concurrently with both galactosyl and xylosyl decorations at the -2 position and with the +1 xylosyl moiety via stacking contacts with $\mathrm{H} 218$ (Fig. 2d). In addition, the arginine residue (R510) stacks with the XyG backbone at the +2 position, and the other three nearby conserved arginine residues (R354, R356, and R513) are strategically located to establish polar and/or stacking interactions with the saccharide at the +3 subsite (Fig. 2e). This molecular strategy of carbohydrate recognition based on arginine residues observed in Xanthomonas GH74 enzymes is a distinguishing feature among $\mathrm{GH}$ families ${ }^{20}$, which typically rely on aromatic $\mathrm{CH}-\pi$ interactions for carbohydrate binding.
The uptake of XyGOs is mediated by TonB-dependent transporters. After the extracellular cleavage of XyG backbone, the following reactions for the deacetylation and breakdown of the released oligosaccharides likely occur at the periplasm, either by the action of free or membrane-anchored enzymes, as indicated by signal peptide analysis and subcellular localization predictions (Supplementary Table 1). Supporting this hypothesis, the knockout of both TonB-dependent transporters (TBDTs) encoded by the XyGUL (XAC1768 and XAC1769) was highly detrimental to $X$. citri growth with XyGOs as carbon source, but not to the growth with a mixture of its monosaccharides, indicating that depolymerization of XyGOs occurs after passing the outer-membrane using specific TBDTs. Individual knockout of these transporters supports a major role for XAC1769 in XyGOs uptake. In the absence of XAC1768, XAC1769 was sufficient to maintain the bacterial growth akin to the wild-type strain using XyGOs as carbon source, but the opposite was not observed. The knockout of XAC1769 impaired the growth in the late log phase, indicating the importance of this transporter as the XyGOs concentration decreases in the culture medium (Supplementary Fig. 5). 
XyGOs deacetylation by Xanthomonas involves a distinctive acetylesterase. Xanthomonas XyGUL harbors a putative esterase gene (XAC1771) initially annotated as sialate 9-O-acetylesterase due to the low similarity with current known carbohydrate esterase families (Supplementary Table 2). The presence of an esterase is in accordance with the fact that fucosylated XyGs from dicotyledons can be acetylated at O6 position of the galactosyl moiety ${ }^{21}$. Acetylation modifies the physical and chemical properties of carbohydrates, limiting enzyme accessibility. Therefore, acetate removal is a key step toward efficient processing of this polysaccharide by downstream glycoside hydrolases.

The Xanthomonas XyGUL esterase, named here XacXaeA (xyloglucan acetylesterase), showed high activity on pNP-acetate and did not accept moieties longer than acetyl as substrates (Supplementary Tables $6-8$, and Supplementary Fig. 6h). It is specific for $\mathrm{O}$-acetylation since it was not capable of cleaving $\mathrm{N}$ acetylated carbohydrates (Supplementary Fig. 7). However, XacXaeA showed activity on a broad range of $\mathrm{O}$-acetylated mono- and disaccharides and did not show a positional preference for acetylated oxygens (Supplementary Fig. 7). As expected, XacXaeA was active towards cell wall extracted xyloglucan oligosaccharides, deacetylating distinct types of structures such as XXLG/XLXG, XXFG, and XLFG (Supplementary Figs. 8 and 9, and Supplementary Table 9).

To get insights into the modular structure and molecular determinants for $O$-acetyl esterase activity, the crystallographic structure of XacXaeA was solved using $\mathrm{Zn}^{2+}$ single-wavelength anomalous dispersion (SAD) (Supplementary Table 4). The catalytic domain displays the SGNH hydrolase fold (Fig. 3a-c) found in several lipases as well as esterases ${ }^{22}$ from families $\mathrm{CE} 2^{23}$, $\mathrm{CE}^{24}, \mathrm{CE}^{25}, \mathrm{CE} 12^{26}$, and $\mathrm{CE} 17^{27}$ (Fig. 3d). However, it is composed of two halves (residues 104-216 and 397-541) due to the insertion of a domain (residues 217-396, named X448 in the CAZy database) in the $a 5-\eta 3$ loop (Fig. 3a-c). Notably, both N(residues 24-103) and C-terminal (residues 542-638) extensions exhibit an antiparallel seven-stranded $\beta$-sandwich fold that did not resemble any known domain at the sequence level. These two iso-structural $\beta$-sandwiches are intimately linked to the esterase core, forming a monolithic structure (Fig. 3b, c). Such structural architecture diverges from carbohydrate esterase (CE) families described in the CAZy database so far.

The active site encompasses the classical catalytic triad (AspHis-Ser) (Fig. 3a, Supplementary Figs. 10a and 11), reminiscent from proteases ${ }^{28,29}$. In addition, it conserves the electropositive oxyanion hole (Supplementary Fig. 10a-c), an ancestral and recurrent feature of enzymes from the GDSL and GDSL-like families of esterases and lipases ${ }^{30,31}$. Interestingly, the catalytic triad is imprinted on a flat surface (Fig. 3c, and Supplementary Fig. 10b) that is uncommon in other known CE families described in the CAZy database 20 (Supplementary Fig. 10d-h). This observation agrees with a lack of selectivity to $O$-acetylated simple sugars since it does not seem to impose steric penalties to any $\mathrm{C} 5$ or $\mathrm{C} 6$ mono- and di-saccharides. Although both $\mathrm{N}$ - and $\mathrm{C}$-terminal $\beta$-sandwich domains are remote to the catalytic center, it is proposed here that they might serve as an extended platform for XyGOs anchoring during acetate removal. Moreover, the internal X448 domain, which was not observed in the crystallographic structure, probably adds another component in the recognition mechanism of complex substrates by this new type of carbohydrate acetylesterase. These functional and structural results allow the classification of XacXaeA as the founding member of the CE20 family.

Xanthomonas enzymatic cascade for XyGOs breakdown. The $\alpha$ 1,2-L-fucosidase activity (EC number 3.2.1.63) was biochemically observed in a GH95 member of the XyGUL (XAC1774), named here XacAfc95. Despite more than one activity has been reported for this family, XacAfc95 appeared to be very specific to L-fucose, which is equivalent to 6-deoxy-L-galactose (Supplementary Tables 6-8, and Supplementary Fig. 12h). Its crystal structure (Supplementary Table 4) conserves the canonical domain architecture of the GH95 family that consists of an N-terminal $\beta$-supersandwich (residues 36-278), $\alpha$-toroidal six-hairpin catalytic domain (residues 349-703), and a C-terminal $\beta$-sandwich (residues 704-790) (Fig. 4a). The catalytic domain is connected to the super sandwich domain by a helix-rich linker (residues 279348) and this multi-domain protomer forms dimers in solution (Supplementary Figs. 13j-l and 14, and Supplementary Table 10). Structural comparisons indicate that the general bases N397/ N399 (carboxylate-activated) and the general acid D690 are conserved in relation to the only two structures available for this family, the $\alpha$-L-galactosidase from B. ovatus ${ }^{32}$ (BACOVA_03438, PDB ID 4UFC, SeqID 42.88\%) and the $\alpha-1,2-L-$ fucosidase from Bifidobacterium bifidum ${ }^{33}$ (BbAfcA, PDB ID 2EAB, SeqID 30.36\%) (Fig. 4b).

Previous comparisons between BACOVA_03438 ( $\alpha$-L-galactosidase) and BbAfcA ( $\alpha-1,2-\mathrm{L}$-fucosidase) led to the suggestion that the only polymorphic position at the -1 subsite would confer specificity to L-galactose or L-fucose, although the authors also pointed that a conclusive inference of the functional relevance of this polymorphic residue is hindered by the very limited structural data available for this family so $\mathrm{far}^{32}$. Based on structural analyses, they proposed that the presence of threonine at this position would allow a hydrogen bond with the L-galactose O6 atom, whereas a histidine would contribute to aliphatic interactions with the L-fucose C6 methyl group. However, XacAfc95, which shares nearly $40 \%$ sequence identity with characterized GH95 $\alpha$-1,2-L-fucosidases involved in XyG depolymerization ${ }^{12,20}$, contains a threonine at the referred position and showed high specificity to L-fucose, contraposing the initial role proposed for this residue as a determinant for Lgalactose preference (Fig. 4b, Supplementary Tables 6-8, and Supplementary Fig. 12h). In addition, the mutation T395H did not result in any change of substrate preference, supporting a less relevant role of this polymorphic position for specificity in the GH95 family (Supplementary Fig. 15a). Besides XacAfc95, another characterized GH95 a-1,2-L-fucosidase (Blon_2335) 34 $^{34}$ conserves a threonine at this polymorphic position, corroborating this hypothesis (Supplementary Fig. 16). These findings point to a more elaborate mechanism of substrate selectivity in the GH95 family that is not limited to direct interactions with the residues forming the -1 subsite.

The subsequent substitution to be cleaved after the $\alpha-1,2-\mathrm{L}$ fucoside removal is a $\beta$-1,2-galactosyl moiety. According to biochemical characterization assays, this step is performed by a $\beta$-galactosidase belonging to the GH35 family (XAC1772) with functional dependence to oligomerization. This enzyme is orthologous to GalD from X. campestris pv. campestris ${ }^{35}$ and forms tetramers in solution (Supplementary Fig. 17a). Structural analysis revealed that the oligomerization interface involves the catalytic domain (TIM-barrel fold, residues 75-458) of one protomer and the accessory $\beta$-sandwich domain (residues 459 585) from the other subunit (Fig. 4c, and Supplementary Fig. 18). Since the functional relevance of oligomerization has not been investigated for this family so far, mutations were designed to address this question. The insertion of an arginine residue (S106R mutation) at the oligomeric interface resulted in stable monomers in solution (Supplementary Fig. 17b), which were devoid of catalytic activity (Supplementary Fig. 15b), supporting a critical role of tetramerization to the function of Xanthomonas GH35 members. 

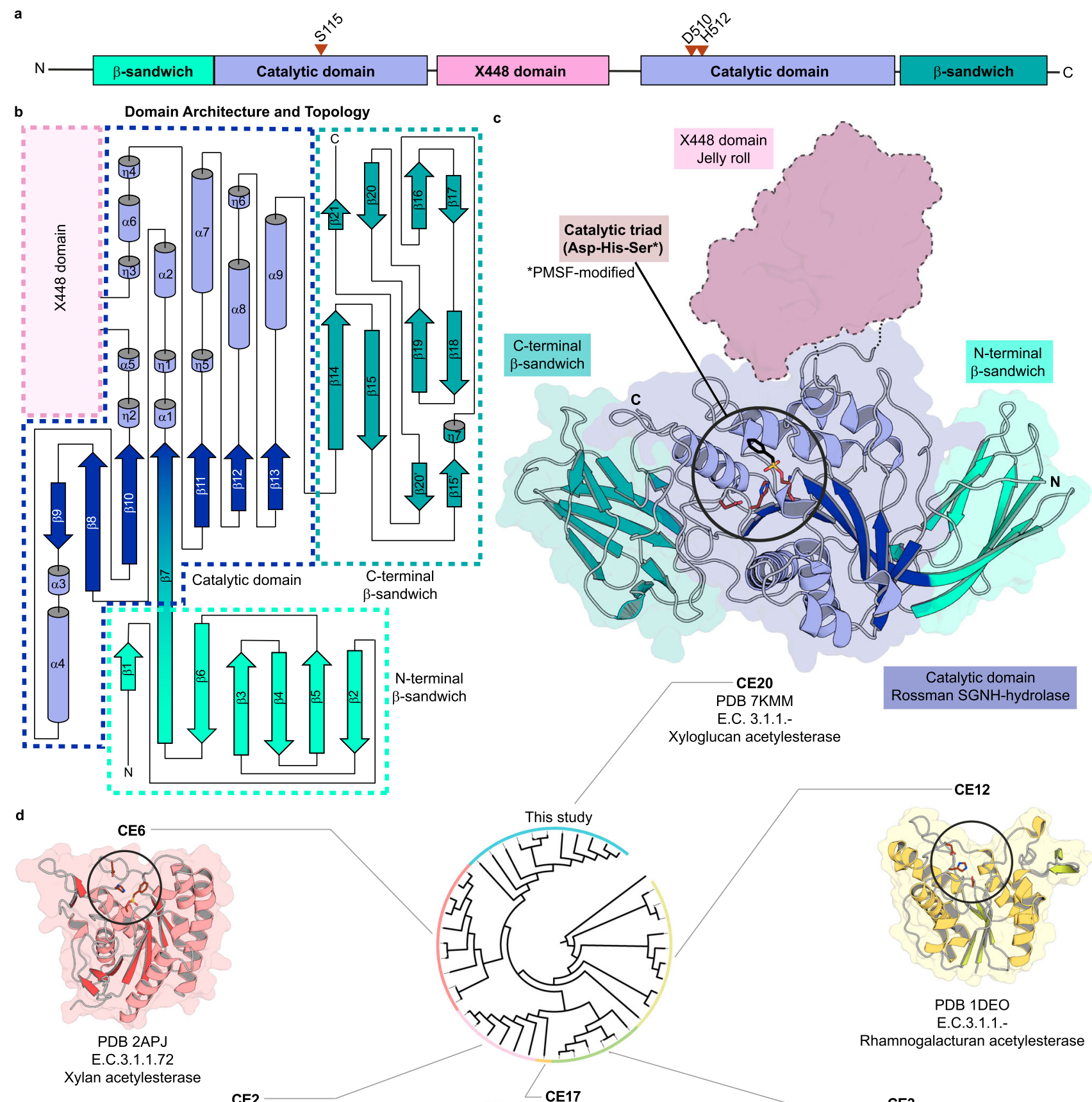

Rhamnogalacturan acetylesterase
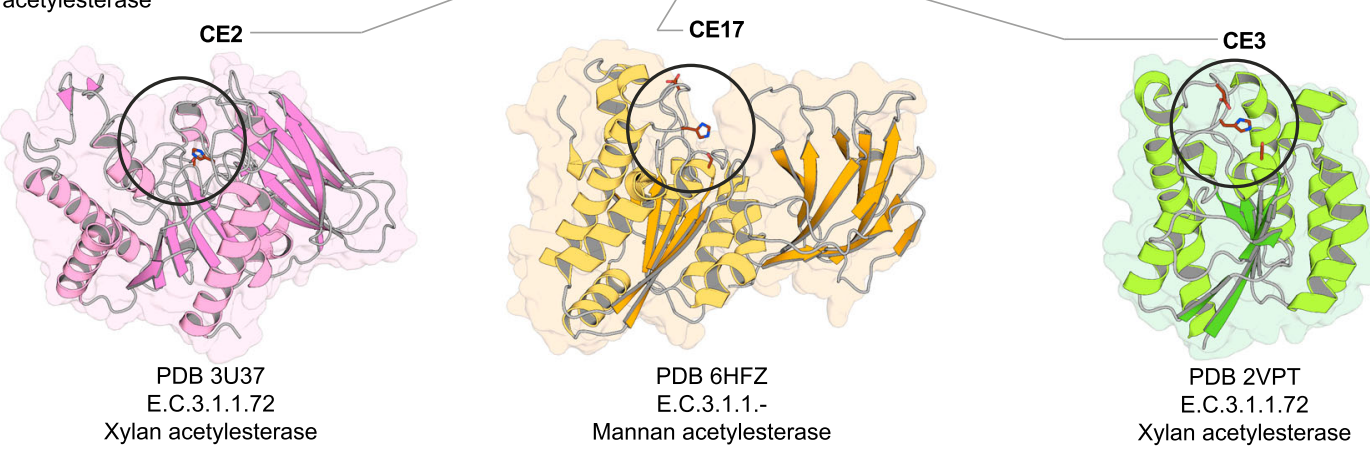

Fig. 3 A distinctive type of carbohydrate acetylesterase. a Domain organization showing the position of catalytic residues (red triangles); $\mathbf{b}$ structural topology and $\mathbf{c}$ crystal structure color-coded according to $\mathbf{a}$. $\mathbf{d}$ Dendrogram of CE families based on phylogenetic analysis of the catalytic domain showing the structure of a representative member of each family. Circles indicate the active site. Catalytic residues (carbon atoms in brown) and PMSF (carbon atoms in black) are shown as sticks. The Xanthomonas acetylesterase, discovered in this study, is the founding member of the CE2O family. 
a $\quad$ GH95 | $\alpha$-L-fucosidase | XacAfc95 | Dime

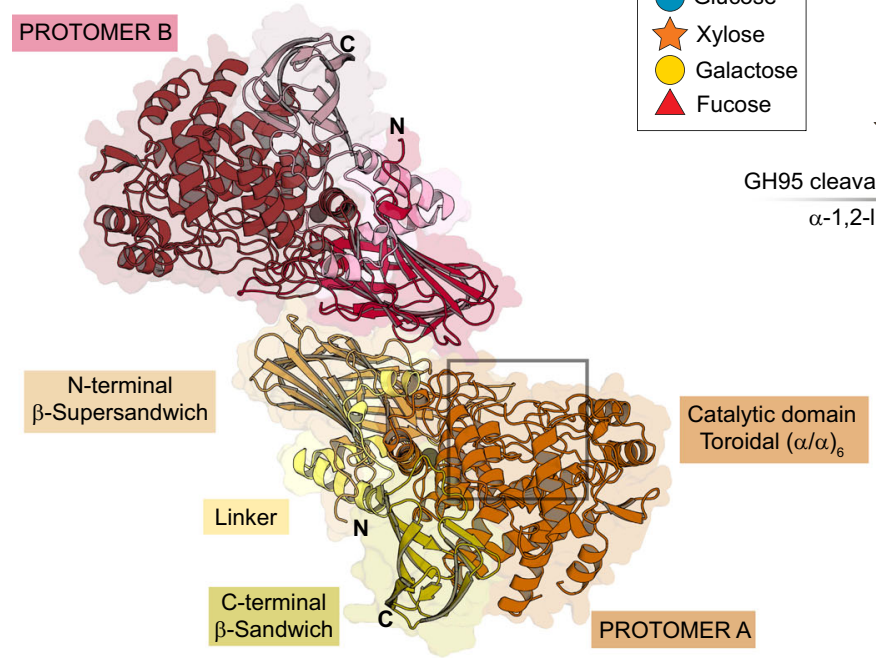

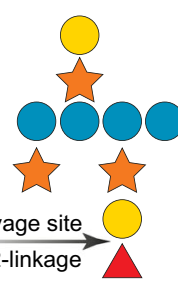

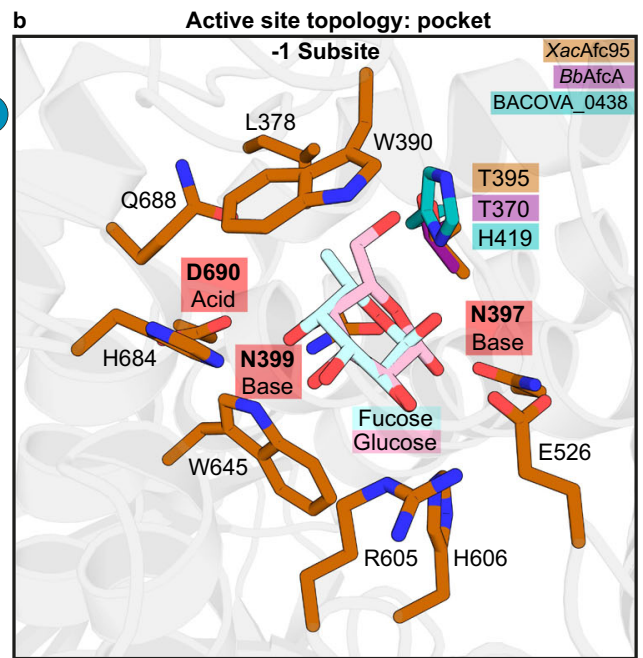

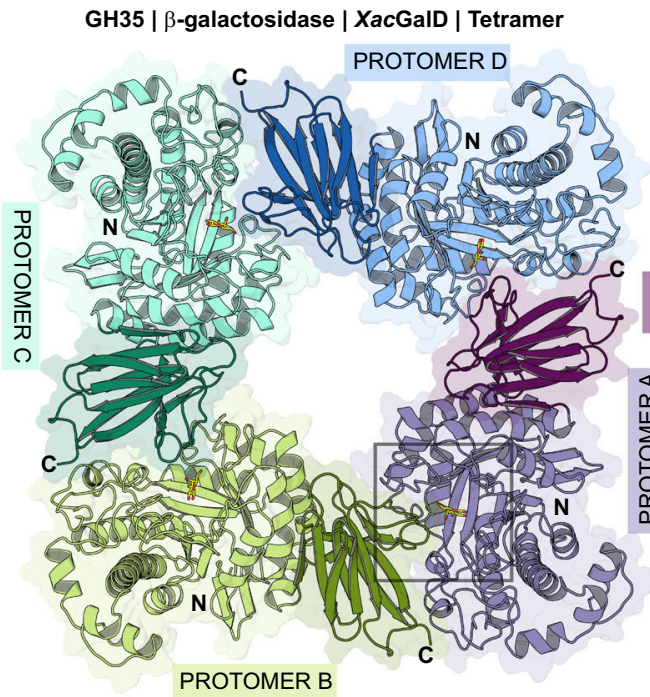

$\mathrm{GH} 35$ cleavage site $\beta$-1,2-linkage

Catalytic domain TIM-Barrel $(\alpha / \beta)_{8}$

GH31 | $\alpha$-xylosidase | XacXyl31 | Monomer

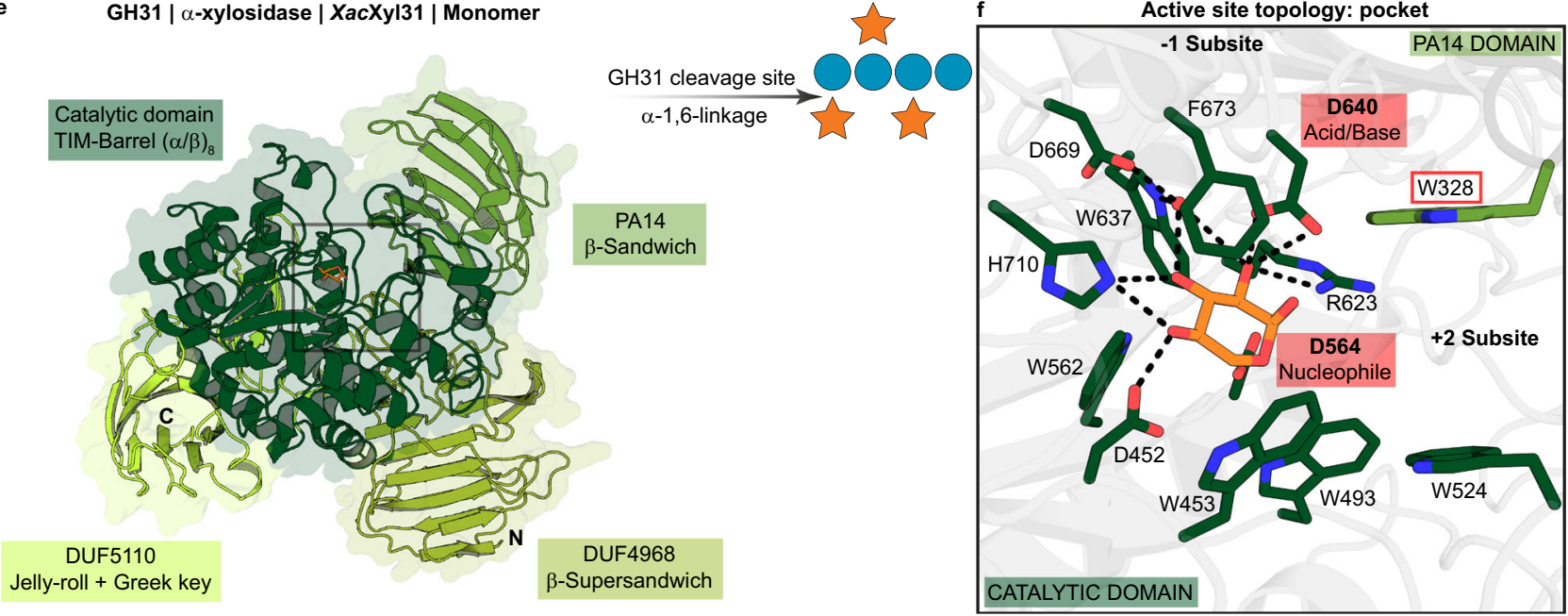

Fig. 4 Molecular basis for the enzymatic cleavage of XyGOs decorations in $\mathbf{X a n t h o m o n a s . ~} \mathbf{a}$, $\mathbf{c}$, e Crystallographic structures of the enzymes that release the substitutions of XyGOs (diagram where blue circle = glucose, orange star = xylose, yellow circle = galactose and red triangle = fucose), highlighting their domains in shades of a specific color defining each protomer or monomer as indicated in the figure. $\mathbf{b}$, $\mathbf{d}, \mathbf{f}$ Amplified view of the enzymes active sites indicated with boxes in panels a, c, e. Residues from the -1 subsite and ligands are shown as sticks (Glucose $=$ pink carbon atoms, Fucose $=$ blue carbon atoms, Galactose = yellow carbon atoms and Xylose=orange carbon atoms). The red outline indicates residues selected for mutational analysis. Catalytic residues are indicated (pink boxes). 
The crystal structure of XacGalD with D-galactose (Supplementary Fig. 19a, b, and Supplementary Tables 4 and 5) revealed that residues in the oligomerization interface also interacts with the saccharide (Fig. 4d), demonstrating that the active-site pocket is completed by residues from the vicinal protomer. Alanine mutation of the residue D562 from the $\beta$-sandwich domain from the neighboring protomer, which interacts directly with the Dgalactose in the active-site pocket (D562A), drastically reduces the enzyme substrate affinity (Supplementary Fig. 15b). Interface analysis of structurally characterized members of the GH35 family, including from archaea, eukaryota, and distinct bacterial phyla (Bacteroidetes, Proteobacteria, and Firmicutes) points out that functionalization by tetramerization is a conserved feature across the phylum Proteobacteria (Supplementary Table 11, and Supplementary Fig. 20). Besides Xanthomonas GH35 enzymes, other representatives from this phylum in the CAZy database, such as CC0788 from Caulobacter vibrioides and CjBgl35A from Cellvibrio japonicus, also form stable tetramers in a similar fashion as XacGalD, which contrasts to the quaternary structures observed in other phyla (Supplementary Table 11).

The next step in the cascade of XyGOs processing involves an a-xylosidase from the GH31 family, according to biochemical analysis of the enzyme encoded by XAC1773 (Supplementary Tables 6-8), named here XacXyl31. The crystal structure of this enzyme (Supplementary Tables 4 and 5) revealed a conserved active-site pocket compared to other bacterial $\alpha$-xylosidases from this family, including the catalytic residues (D564 as nucleophile and D640 as acid/base) and the aromatic residue (W453). The latter introduces a steric barrier to C6 saccharides, favoring only the accommodation of $C 5$ sugars such as xylose at the -1 subsite ${ }^{36}$ (Fig. 4e, f and Supplementary Fig. 19c, d).

Another conserved feature of GH31 a-xylosidases specific to XyGOs, present in XacXyl31, is the four-domain arrangement consisting of a central TIM-barrel catalytic domain (residues 392757 ) and three all- $\beta$ fold accessory domains (DUF4968, residues 38-219 and 372-391; DUF5110, residues 758-957; and PA14,

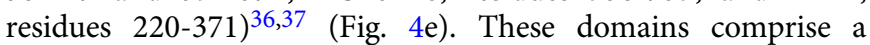
monolithic tertiary structure and do not establish oligomeric contacts in any of the GH31 members structurally characterized so far, including the enzyme reported here (Supplementary Fig. 13g-i, and Supplementary Table 10). Among the four accessory domains, the PA14 is the only participating in the active site interface (Fig. 4e, f) ${ }^{37-39}$. This domain introduces an aromatic platform (W328) at the +2 subsite that along with W524 from the TIM-barrel catalytic domain establishes stacking interactions with XyGOs backbone (Fig. 4f) $37,40,41$. The residue W328 is highly conserved in GH31 a-xylosidases and the mutation W328A was detrimental to the catalytic activity (Supplementary Fig. 15c), supporting the importance of this region for $\alpha$-xylosidase activity in the GH31 family.

As previously described in C. japonicus, GH31 a-xylosidases active on XyGOs may cleave specifically $\alpha(1,6)-\mathrm{Xyl} p$ moieties appended to the non-reducing end of the backbone, requiring the cooperation of XyGOs-specific $\beta$-glucosidases to complete XyGOs depolymerization ${ }^{37,42}$. XacXyl31 also presented this specificity (Supplementary Fig. 21), but no $\beta$-glucosidase-encoding gene was found in the Xanthomonas XyGUL.

On the other hand, five potential $\beta$-glucosidases are present in the $X$. citri genome, all belonging to the polyspecific GH3 family. The heterologous expression and biochemical characterization of these enzymes revealed that two of them are $\beta$-xylosidases (XacXyl3A$\mathrm{XAC} 3076$ and XacXyl3B-XAC4231) and the other three are $\beta$-glucosidases (XacBgl3A-XAC1448, XacBgl3B-XAC1793, and XacBgl3C-XAC3869) (Supplementary Tables 6 and 7).

These three $\beta$-glucosidases were expressed in the presence of XyGOs with higher expression levels of $\mathrm{XacBgl} 3 \mathrm{C}$ and $\mathrm{XacBgl} 3 \mathrm{~B}$ compared to XacBgl3A (Supplementary Table 12) (see section below). In addition, all three enzymes were capable of releasing the non-reducing glucosyl moiety from the XyG-derived oligosaccharides GXXG and GXG, which are the products of XacXyl31 using XXXG and XXG as substrates, respectively (Supplementary Figs. 21 and 22).

XacBgl3B displayed the highest activity on GXXG and GXG substrates and is predicted to be an outer membrane associated protein, probably exposed to the periplasm (Supplementary Fig. 22, and Supplementary Table 1). XacBgl3A, although predicted to be periplasmic, seems to be more specific to cleave $\beta$-1,3-glucooligosaccharides instead of XyGOs-derived $\beta$-1,4glucooligosaccharides (Supplementary Figs. 23 and 24, and Supplementary Tables 1 and 8). XacBgl3C displayed a more generalist substrate profile and is predicted to be cytoplasmic, indicating that it might support the final steps of glucooligosaccharides cleavage coming from different sources (Supplementary Figs. 23 and 24, and Supplementary Tables 1 and 8).

Together, these findings point to $\mathrm{XacBgl} 3 \mathrm{~B}$ as being the major $\beta$-glucosidase to alternate with $\mathrm{XacXyl31}$ on the breakdown of XyGOs intermediates until reaching the final substrate $\beta-1,4$ glucobiose. At this last step, $\mathrm{XacBgl} 3 \mathrm{~B}$ seems also to play an important role, since it was the most efficient on $\beta$-1,4-glucobiose cleavage, compared to XacBgl3A and XacBgl3C (Supplementary Fig. 24, and Supplementary Table 8).

XyG depolymerization products activate the expression of virulence factors. The presence of XyGULs in both mesophyllic and vascular Xanthomonas pathogens indicates that XyG processing might play a role in bacterial colonization, pathogenicity and/or survival in host plants. To explore this hypothesis, the global gene expression profile of $X$. citri was assessed in the presence of XyGOs, which resulted in 276 differentially expressed genes among its 4281 protein coding sequences (Fig. 5a, and Supplementary Data 1).

As expected, the presence of XyGOs in the medium increased the expression of XyGUL genes (XAC1768-XAC1774), downstream genes related to xylose metabolism (XAC1775 and XAC1776) and an MFS transporter (XAC1777), supporting the relevance of this system for XyG processing in vivo (Fig. 5b). Moreover, these oligosaccharides upregulated two conserved GH43 encoding genes (XAC1275 and XAC4183) that are not in the vicinity of the XyGUL (Supplementary Fig. 2 and Supplementary Data 1). The enzyme encoded by XAC4183, named here XacAbf43A, belongs to an underexplored subfamily, the GH43_9, with only one reported member characterized so far with a weak arabinofuranosidase activity ${ }^{43}$. To evaluate this activity in the Xanthomonas member of this subfamily, XacAbf43A was produced, and biochemical assays confirmed its $\alpha$-L-arabinofuranosidase activity (Supplementary Tables 6 and 7). The recombinant production of XAC1275 did not yield a soluble and stable protein; however, it displays $37 \%$ of sequence identity with the GH43_12 arabinofuranosidase from the $B$. ovatus $\mathrm{XyGUL}^{40}$, pointing to a potential similar functional specificity. The fact that these GH43 genes are conserved across Xanthomonas bacteria, positively regulated by $\mathrm{XyGOs}$ and functionally related to orthologues present in Bacteroides XyGUL indicate that Xanthomonas could act on both types of XyGs, fucogalactoxyloglucans (present in plants from the Rosids clade ${ }^{44}$ ) and arabinoxyloglucans (present in plants from the Asterids clade, in the Solanaceae and Oleaceae families ${ }^{45}$ ).

The sensing of XyG depolymerization products by Xanthomonas also modulated other aspects of the bacterial metabolism (Fig. 5c). While repressing genes related to chemotaxis and flagellar motility, XyGOs stimulated processes that play essential 
a

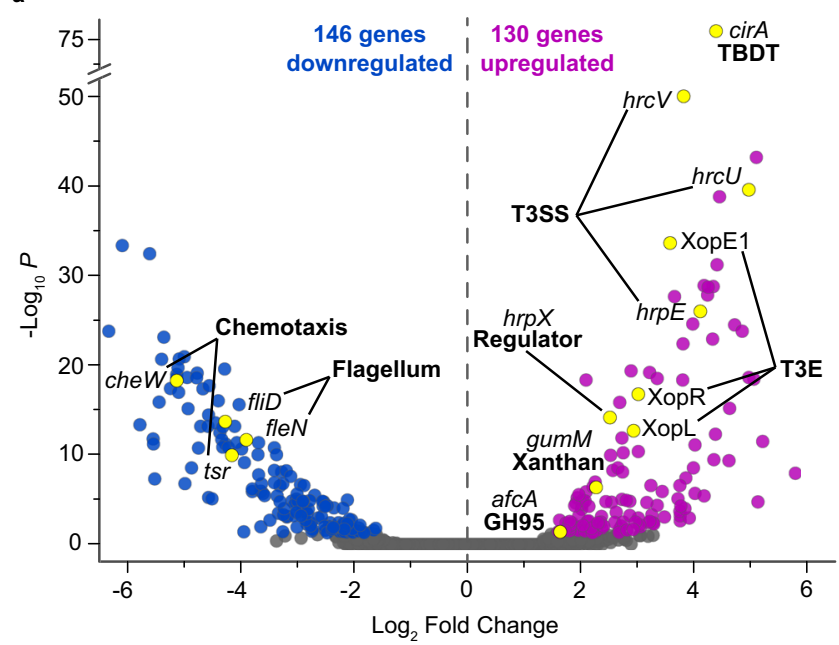

b

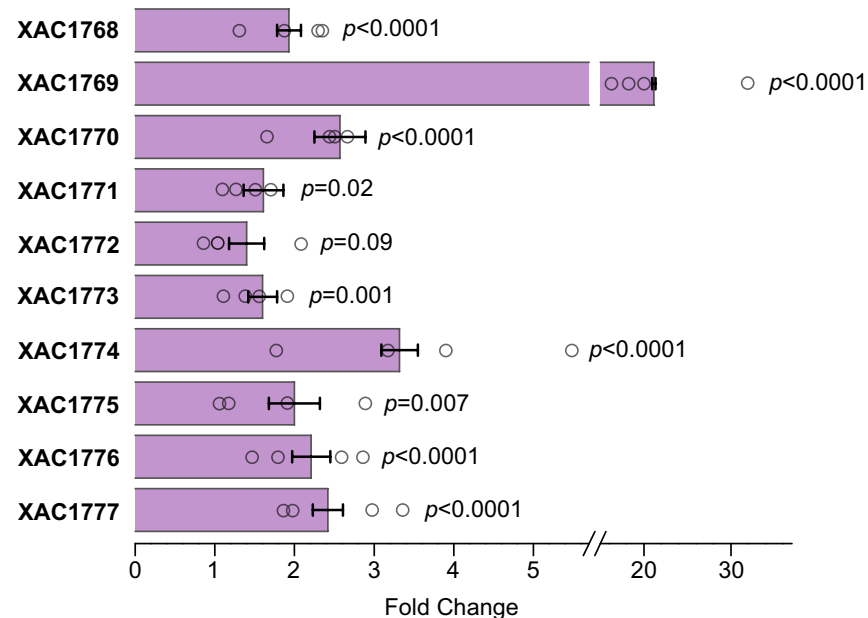

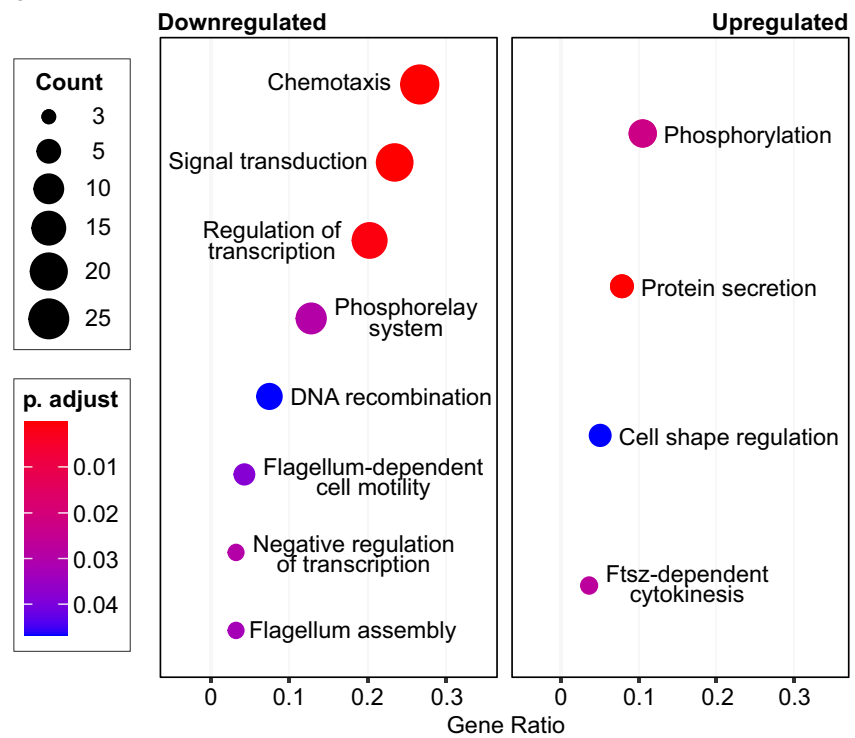

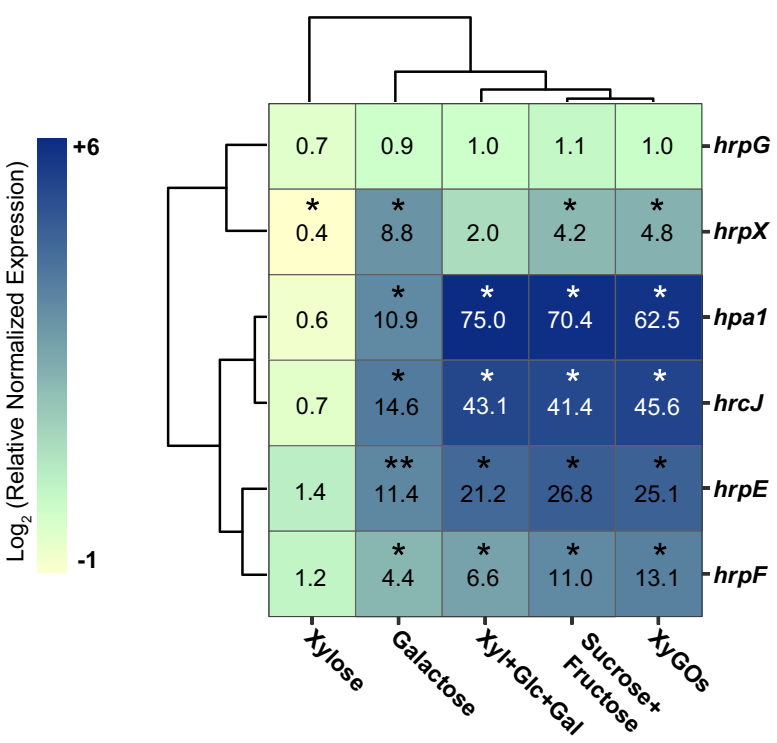

Fig. 5 Transcriptional responses to XyGOs in X. citri. a Volcano plot of RNA-seq data highlighting differentially expressed genes (DEGs; blue circles = downregulated and purple circles $=$ upregulated). In yellow are DEGs acting on processes important for virulence or XyG utilization. Genes were considered differentially expressed according to Wald test implemented in DESeq2. p-values were adjusted for multiple tests using the BenjaminiHochberg (BH) method implemented in DESeq2. Thresholds: $p$-adjusted $<0.05$ and $\mid \log _{2}$ Fold Change $>1$. b Transcription levels of XyGUL genes, downstream genes encoding xylose metabolism and MFS transporter in RNA-seq experiments. Data shown as mean \pm SE from four biologically independent experiments $(n=4)$ (open circles). Threshold: BH adjusted $p$-value $<0.05$. c Gene ontology $(G O)$ enrichment analysis of DEGs. Circles size and color represent the counts and BH adjusted $p$-values, respectively, according to the legend at the left. Gene ratio is the number of DEGs related to a GO term divided by the total number of annotated DEGs. Categories were considered enriched based on hypergeometric test, implemented in the clusterProfiler 3.14.3R/Bioconductor enrich function ${ }^{60}$. $\mathbf{d}$ Clustered heat map of RT-qPCR data of hrp genes color-coded according to the log $2(-1$ to +6$)$ of the mean normalized expression relative to the control (numbers inside the boxes). Two-tailed $t$ test: ${ }^{\star}, p<0.001 ;{ }^{\star \star} ; p<0.0001 ; \mathrm{Xyl}=\mathrm{xylose}, \mathrm{Glc}=$ glucose and $\mathrm{Gal}=$ galactose. See details in Supplementary Fig. 25.

roles in the early stages of plant infection ${ }^{46}$, including xanthan gum biosynthesis and secretion of type III protein effectors into the host cells. Seven gum genes (XAC2574-XAC2580), the entire T3SS cluster (XAC0393-XAC0417), and 14 effector protein genes were up-regulated by XyGOs (Supplementary Data 1, and Supplementary Table 13), indicating an unprecedented role of $\mathrm{XyG}$ depolymerization products in bacterial virulence. As XyGs have a complex structure, RT-qPCR assays were performed to assess whether the activation of T3SS genes depends on the structure of XyGOs or its basic core constituents, i.e., glucose, galactose, and xylose. In these experiments, the mixed-sugar condition (glucose plus galactose and xylose) activated the expression of T3SS genes akin to the XyGOs condition, showing that the signaling effect of XyGOs relies on its monomers and not on its complex structure (Fig. 5d, and Supplementary Fig. 25).

To gather insight into the signaling pathway assessed by XyG depolymerization products, we also evaluated the expression of master regulators of T3SS expression, $h r p G$ and $h r p X^{47}$. Although $h r p G$ was not activated in any of the tested conditions, the expression of $h r p X$ increased in the presence of galactose, XyGOs and sugar mix, being maximal in the galactose condition (Fig. 5d, and Supplementary Fig. 25). This result shows that galactose is sufficient for the transcriptional activation of $h r p X$. However, for all T3SS genes analyzed, the transcription was further stimulated when galactose was supplemented with glucose and xylose, indicating that a second signal coming from other XyG 
components boosts the expression of T3SS genes (Fig. 5d, and Supplementary Fig. 25).

The combination of the monosaccharides mimicking the XyG breakdown products can also be achieved by the synchronized depolymerization of other plant polysaccharides and is as potent as the combination of sucrose and fructose in stimulating the T3SS expression in vitro (Supplementary Fig. 25), supporting that Xanthomonas relies on multiple and redundant sources of signals to trigger virulence and modulate host responses. Therefore, to confirm this redundancy hypothesis, we evaluated whether $X$. citri pv. citri would maintain its virulence even with the knockout of XyGUL genes (TBDTs and xyloglucanase) or an adjacent MFS transporter (Supplementary Fig. 26). As envisaged, the wild-type phenotype was preserved in these mutants, supporting that the activation of T3SS by XyG depolymerization products is probably compensated via functional redundancy or via alternative pathways for virulence activation, especially in the case of $\triangle \mathrm{XAC1768-}$ 69 deletion. The in vitro growth of this mutant in minimal medium containing XyGOs was severely reduced, indicating that the lack of XAC1768-69 genes impairs the uptake of these oligosaccharides (Supplementary Fig. 5a).

The deletion of the inner-membrane MFS transporter gene (XAC1777) did not affect the bacterial growth using either XyGOs or its basic components as primary carbon source, supporting that at least one of the other 40 MFS transporters encoded by X. citri genome compensate its absence (Supplementary Fig. 5, and Supplementary Table 14). Notably, the strain lacking the GH74 xyloglucanase ( $\triangle \mathrm{XAC1770)}$ displayed a XyG depolymerization halo similar to the wild-type strain, suggesting that another endo- $\beta$-1,4-glucanase would be functionally redundant to the XyGUL GH74 enzyme (Supplementary Fig. 27). A search for endo- $\beta$-1,4-glucanases in $X$. citri genome resulted in one GH9 member (XAC2522, XacEgl9), one GH8 member (XAC3516, $\mathrm{XacCel}^{48}$ ), and five putative GH5 glucanases (XAC0612-subfamily GH5_1, XacEngXCA; XAC0028-subfamily GH5_5, XacEgl5A; XAC0029—subfamily GH5_5, XacEgl5B; XAC0030-subfamily GH5_5, XacEgl5C; and XAC0346-not yet assigned to a subfamily $)^{49}$. The recombinant production and activity assays of these enzymes revealed that only XacEgl9, XacEgl5B, and XacEngXCA are able to cleave XyG, with XacEgl9 showing the highest specific activity on this polysaccharide (Supplementary Table 15). This result indicates that these glucanases might compensate the absence of the GH74 xyloglucanase in the $\triangle \mathrm{XAC1770}$ mutant. Characterization of XacEgl9 revealed kinetics parameters on XyG akin to those found for XacXeg74 (Supplementary Table 8, and Supplementary Figs. $6 \mathrm{~g}$ and $12 \mathrm{i}$ ), supporting the role of other endo- $\beta$ 1,4-glucanases in the XyG cleavage in strains lacking the GH74 enzyme.

\section{Discussion}

Here, we show that most Xanthomonas species, a highly diverse bacterial genus that infects hundreds of plants, have an intricate enzymatic toolbox to break down XyGs. The Xanthomonas XyGUL encodes oligomeric and multi-modular glycoside hydrolases (GH74 xyloglucanase, GH31 a-xylosidase, GH35 $\beta$-galactosidase and GH95 $\alpha$-L-fucosidase) and a distinguishing carbohydrate acetylesterase with no significant similarity with known CAZy families and not present in any similar XyGUL characterized so far 5,6,12 (Figs. 1 and 6). This novel acetylesterase, $\mathrm{XacXaeA}$, is the founding member of the CE20 family.

Transcriptomic, biochemical and gene deletion analyses revealed that the Xanthomonas XyGUL is complemented by other conserved CAZyme genes encoding GH9 and GH5 enzymes with xyloglucanase activity, GH3 $\beta$-glucosidases and GH43 arabinofuranosidases. The GH9 (XacEgl9) and GH5 (XacEgl5B) enzymes serve as redundancy components to the pivotal xyloglucanase activity that initiate $\mathrm{XyG}$ breakdown and the GH43 enzymes, such as XacAbf43A, confer the ability to these bacteria to cleave both fucogalactoxyloglucans ${ }^{44}$ and arabinoxyloglucans ${ }^{45}$. In the final steps of XyG processing, the GH31 a-xylosidase (XacXyl31) acts coordinately with a XyGOs-active $\beta$-1,4-glucosidase $(\mathrm{XacBgl} 3 \mathrm{~B})$ at the non-reducing end of XyGOs, cycling between xylosyl side chain cleavage and glucosyl main chain removal, as previously observed in the saprophyte C. japonicus ${ }^{5,42}$. A difference is that $C$. japonicus displays a $\beta$-glucosidase with the highest efficiency toward the intermediate GXXG and another one with the highest efficiency on $\beta$-1,4-glucobiose, whereas in $X$. citri the enzyme XacBgl3B seems to play both roles. These observations highlight that microbial systems, according to their ecological niches, have evolved singular and equally complex molecular strategies to cope with the structural and chemical diversity of XyGs.

From a mechanistic point of view, the enzymes encoded by Xanthomonas XyGUL also show distinguishing properties compared to other characterized homologs. The GH74 enzyme harbors an unusual arginine-based mechanism of substrate recognition, contrasting to the canonical strategy in the $\mathrm{GH}$ families based on aromatic $\mathrm{CH}-\pi$ interactions ${ }^{50}$ (Fig. 2). The xyloglucan acetylesterase features an unprecedented molecular architecture with two iso- $\beta$ domains at both $\mathrm{N}$ - and $\mathrm{C}$-termini and the insertion of an uncharacterized domain in the middle of the catalytic core (Fig. 3). The GH95 member demonstrates that substrate specificity in this family does not involve polymorphic positions in the -1 subsite, pointing to the relevance of indirect interactions in determining selectivity. In addition, the role of ancillary domains and oligomerization is prominent in the function of Xanthomonas XyGUL enzymes (Fig. 4).

Notably, the XyG depolymerization products play a role in the activation of multiple genes related to virulence in Xanthomonas spp., including those encoding effector proteins and the T3SS that inject these virulence factors into the host cells (Fig. 5). In the genus Xanthomonas, the activation of T3SS expression by carbohydrates such as sucrose and fructose in $X$. campestris $\mathrm{pv}$. campestris and $X$. campestris pv. vesicatoria ${ }^{51,52}$, and xylose in $X$. oryzae pv. oryzae ${ }^{53-55}$, has been previously demonstrated. However, the role of galactose in this process and XyG depolymerization as a source of T3SS inducers, are novel components in the complex regulatory mechanisms of virulence in these pathogens.

Our results demonstrate that galactose from XyG depolymerization activates the transcription of $h r p X$, a master regulator of T3SS expression (Fig. 6). The role of galactose as an inducer of $h r p X$ gene correlates with a previous study in $X$. oryzae showing that activation of $h r p X$ expression is mediated by a regulator of galactose metabolism, termed $\mathrm{GamR}^{56}$ (XAC1767). In addition to galactose activation, a second signal from xylose, acting after the $h r p X$ expression, likely contributes to the expression of T3SS genes. Similarly to that proposed for $X$. oryzae, the induction of T3SS by xylose might be associated with a post-transcriptional mechanism that suppresses HrpX proteolysis ${ }^{54}$. Based on these observations, we propose that during $\mathrm{XyG}$ depolymerization, the released galactose activates the transcription of $h r p X$, likely by modulating the GamR activity, whereas the released xylose suppresses HrpX degradation, thus promoting the activation of T3SS genes and other HrpX-mediated processes (Fig. 6). It is noteworthy that most Xanthomonas bacteria are equipped with other CAZymes and polysaccharide utilization loci specialized in depolymerizing other hemicelluloses ${ }^{57,58}$ and pectins ${ }^{59}$, which can also generate xylose and galactose, adding more layers of complexity in the modulation of virulence and pathogenesis mediated by host carbohydrate processing in these phytopathogens. 


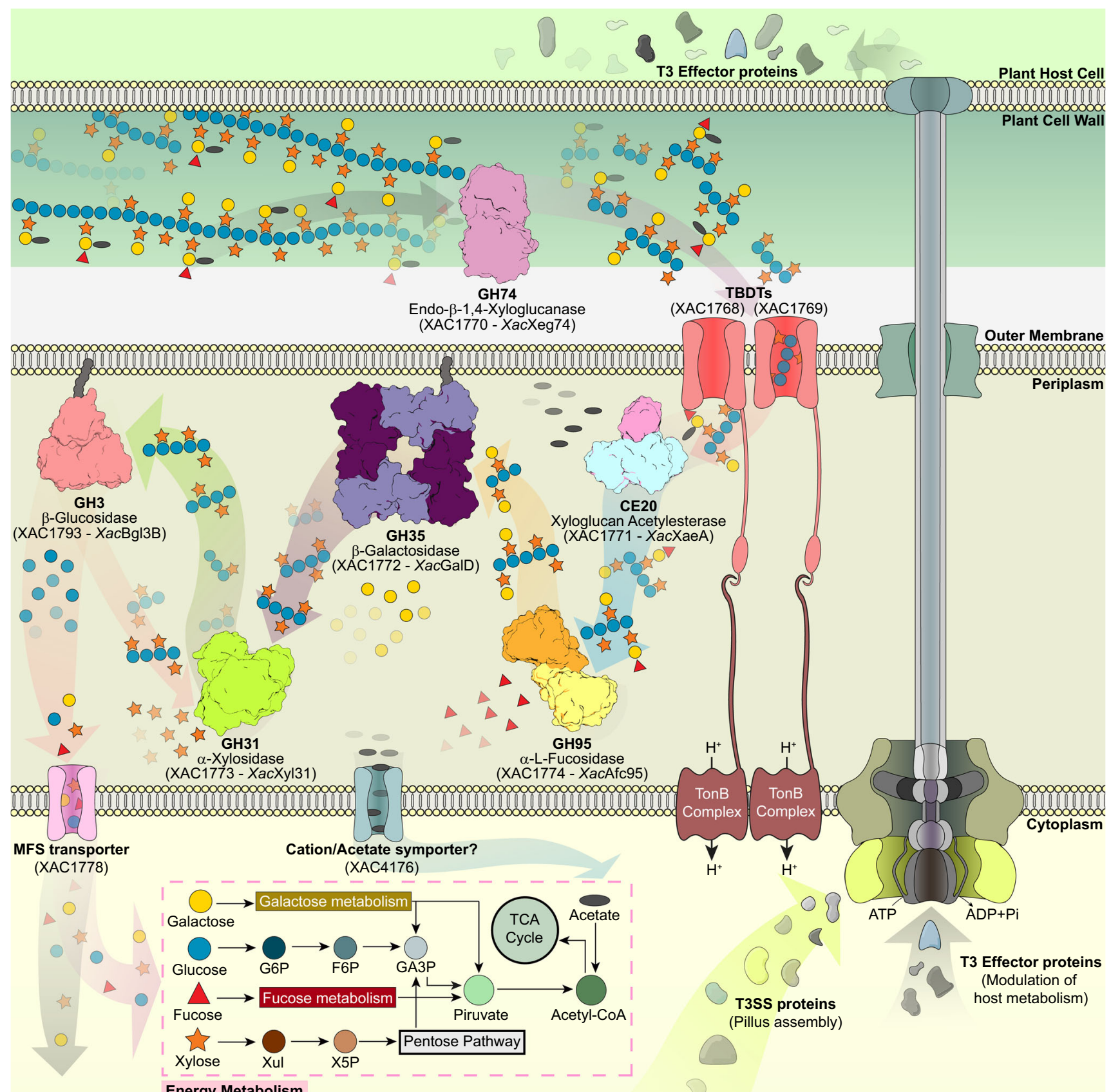

\section{Xylose activation pathway}

T3SS cascade activation

Galactose activation pathway
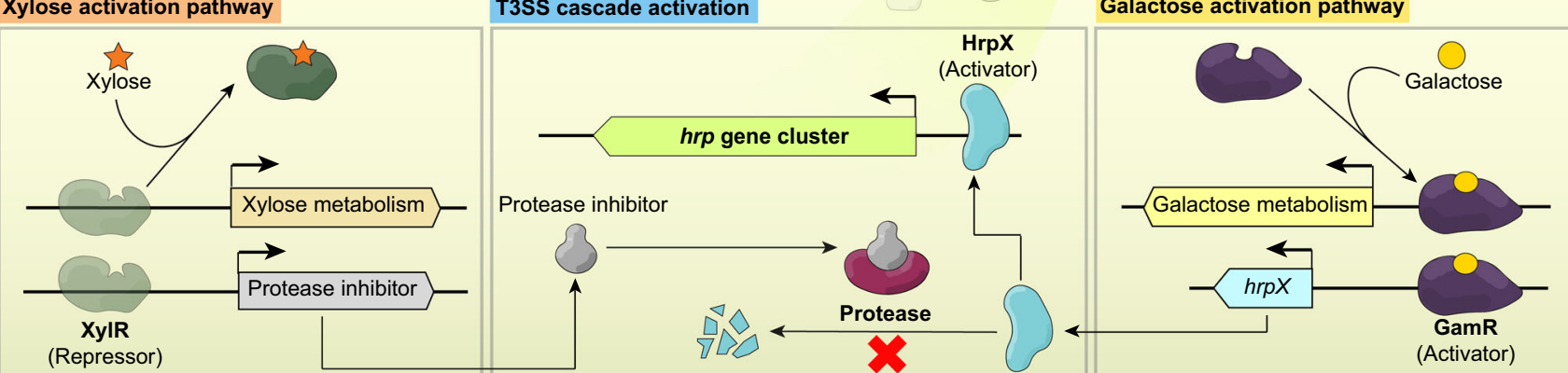

Fig. $\mathbf{6}$ XyG enzymatic breakdown and the role of released products in the metabolism and virulence of $\mathbf{X}$. citri. Representation of the pathogen-host cell interface highlighting how XyG is progressively depolymerized by Xanthomonas enzymes and internalized by transmembrane transporters. In this bacterium, monosaccharides released from XyG induce the expression of multiple genes, including the T3SS machinery via mechanisms suggested based on transcriptional data and literature ${ }^{54-56}$ (details in Supplementary Fig. 28, and Supplementary Table 1). T3, Type III; T3SS, Type III secretion system; TBDTs, TonB-dependent transporters; MFS, major facilitator superfamily transporter; G6P, D-glucose-6-phosphate; F6P, D-fructose-6-phosphate, GA3P, D-glyceraldehyde 3-phosphate; Xul, D-xylulose; X5P, D-xylulose 5-phosphate; and TCA, Tricarboxylic Acid cycle. 
In conclusion, this work provides an in-depth understanding of the multi-enzymatic system employed by plant-associated bacteria for XyG depolymerization, and also establishes a novel component in the regulatory mechanisms of virulence and pathogenesis in Xanthomonas.

\section{Methods}

Phylogenetic analysis. Phylogenetic analysis of Xanthomonas species was performed based on a set of 92 single-copy core genes according to the UBCG pipeline $3.0^{61}$. Individual proteins were aligned separately using MAFFT 7.299b and then concatenated. Phylogenetic analysis was inferred using RAxML 8.2.0 and the PROTGAMMAWAG model with 1,000 bootstrap replicates. Applying a similar protocol, phylogenetic analysis of carbohydrate acetylesterases was perfomed with sequences of the catalytic domain of characterized enzymes available at the CAZy database (http://www.cazy.org/) including homologous sequences of XacXaeA.

Molecular cloning and site-directed mutagenesis. The nucleotide sequence encoding the XyGUL and accessory enzymes were amplified from the genomic DNA of X. citri pv. citri 306 strain or X. campestris pv. campestris ATCC 33913 using standard methods (Supplementary Table 16) and confirmed by Sanger sequencing. PCR-amplified gene fragments were cloned into the pET28a and pETM11 vectors. The mutants were prepared using the QuikChange II XL SiteDirected Mutagenesis Kit (Agilent) (Supplementary Table 17).

Protein expression and purification. The proteins were expressed in Escherichia coli strains as described in the Supplementary Table 18. Proteins were purified by two chromatographic steps as detailed in the Supplementary Table 19. Purified samples were analyzed by SDS-PAGE and dynamic light scattering (DLS) in a Malvern ZetaSizer Nano series Nano-ZS (model ZEN3600) instrument (Malvern Zetasizer). DLS data were collected and analyzed with Zetasizer (7.12) software to evaluate sample homogeneity.

Analytical hydrodynamic analysis. Size-exclusion chromatography coupled with multi-angle light scattering (SEC-MALS) experiments were performed at $25^{\circ} \mathrm{C}$ using a triple-angle static light scattering detector miniDAWN ${ }^{\mathrm{sm}}$ TREOS and Optilab T-rEX refractive index monitor (Wyatt Technology) coupled to an ÄKTA fast protein liquid chromatography system (GE Healthcare) with a Superdex 200 HR 10/300 GL analytical size-exclusion column (GE Healthcare) ${ }^{62} .250 \mu \mathrm{L}$ from purified wild-type $X a c G a l D$ and mutant S106R at $50 \mu \mathrm{mol} \mathrm{L}^{-1}$ were injected separately into the column

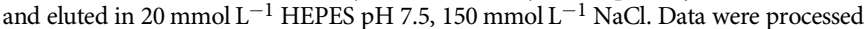
using the ASTRA V software 6.0 (Wyatt Technology).

Small-angle X-ray scattering (SAXS) data collection was performed with protein samples at different concentrations $\left(1,3,5\right.$, and $\left.10 \mathrm{mg} \mathrm{mL}^{-1}\right)$ at the D01A-SAXS2 beamline at the Brazilian Synchrotron Light Laboratory (LNLS-CNPEM, Campinas, Brazil), using a CCD-Mar165 detector and fit2D software v. 18. Data were processed and analyzed with the ATSAS package $4.8 .6^{63}$ using the programs GNOM v. 5.0, DAMMIN v. 5.3, DAMAVER v. 5.0, CRYSOL v. 2.8.3, and SUPCOMB v. 2.3. SAXSMoW server ${ }^{64}$ was used for protein molecular weight determination, and oligomeric interface interaction energy was calculated using the PDBePISA server 65

\section{Protein crystallization, X-ray data collection, and structure determination} Proteins were crystallized by the vapor diffusion method (Supplementary Table 20). Diffraction data were acquired under cryogenic conditions at the MX2 beamline from the Brazilian Synchrotron Light Laboratory (LNLS-CNPEM, Campinas, Brazil) using a Pilatus 2M detector (Dectris) and MXCuBE 2 (Qt4) software or at the BL9-2 beamline from the Stanford Synchrotron Radiation Lightsource (SSRL-SLAC, Menlo Park, USA) using a Pilatus 6M detector (Dectris) and BluIce 4.0 software. Data were indexed, integrated and scaled using the XDS package v. Jan 31st 2020 Built 2020041766. XccXeg74, XacGalD, XacXyl31 and XacAfc95 structures were solved by molecular replacement method using the PHASER software from PHENIX package dev- $3139^{67}$ and the atomic coordinates from homologous proteins $2 \mathrm{CN} 2^{68}, 4 \mathrm{D} 1 \mathrm{I}^{5}, 2 \mathrm{XVG}^{37}$ and $4 \mathrm{UFC}^{32}$, respectively. Structure of XacXaeA was solved by zinc SAD using the programs SHELXC/D/E from CCP4i package 7.0.023 ${ }^{69}$. The initial model of XacXaeA was obtained with AutoBuild Wizard from the PHENIX package dev-313970 and further refined iteratively with COOT $0.8 .9^{71}$ and PHENIX refine dev-3139 programs. Structure validation was carried out with the Molprobity server ${ }^{72}$. Metal-binding sites validation was performed with the CheckMyMetal server ${ }^{73-75}$. Carbohydrate complexes structures were evaluated using Privateer software from CCP4i2 package 1.0.2 revision $5710^{76}$ and figures were generated using Pymol v. 2.3 or 1.3. Data collection, processing and analyses are summarized in Supplementary Tables 4 and 5.

Glycoside hydrolase assays. XyGUL and accessory GHs activities were evaluated against several synthetic and natural substrates as described in Supplementary Table 21. The enzyme amount and reaction time for enzyme assays were determined based on linearity tests previously performed. Spectrophotometric data were collected in an Infinite 200 PRO microplate reader (Tecan) using the iControl 1.10.4.0 software (Tecan). Kinetic parameters were determined from substrate saturation curves using the OriginPro 8.1 software. All enzyme assays consist of at least three independent experiments.

C. langsdorffii XyG was extracted from the cotyledons powder by washing three times with $80 \%$ ethanol solution at $80^{\circ} \mathrm{C}$ for $10 \mathrm{~min}$ to remove low molecular weight carbohydrates. Polysaccharides were extracted by resuspending in water the ethanol-insoluble portion of the material and incubating for $8 \mathrm{~h}$ at $80^{\circ} \mathrm{C}$ under constant agitation. The mixture was filtrated, added three volumes of ethanol and centrifuged $(12,000 \mathrm{~g}$ for $15 \mathrm{~min})$. The supernatant was discarded and the pellet dried at $80^{\circ} \mathrm{C}^{77}$. The XyGOs released from C. langsdorffii XyG by XccXeg74 enzyme were analyzed by the HPAEC-PAD system (Dionex) equipped with the CarboPac PA100 analytical column (Dionex). Identification of XyGOs was performed by MALDI-TOF on a Bruker Autoflex MALDI-TOF mass spectrometer (Bruker Daltonics) in reflectron positive mode with a $19 \mathrm{kV}$ voltage and covered mass within the $m / z$ values of $700-3500$.

The activity of the enzymes $\mathrm{XacBglA}, \mathrm{XacBglB}$, and $\mathrm{XacBglC}$ on $\mathrm{XyGO}$ s was investigated after the removal of the xylosyl moiety at the non-reducing end glucosyl residue in XyGOs by the $\beta$-xylosidase $\mathrm{XacXyl} 31$. Reactions with $\mathrm{XacXyl31}$ $\left(0.1 \mathrm{mg} \mathrm{mL}^{-1}\right)$ were performed using $10 \mathrm{mg} \mathrm{mL}^{-1}$ XyGOs (Megazyme O-X3G4) incubated at $45^{\circ} \mathrm{C}$ and $\mathrm{pH}$ 6.5. After $60 \mathrm{~min}$, the reactions were stopped by boiling for $5 \mathrm{~min}$ and the final products (GXXG and GXG) were used for activity assays with the three $\beta$-glucosidases. $\mathrm{XacBglA}, \mathrm{XacBglB}$, and $\mathrm{XacBglC}$ reactions consisted of adding $0.1 \mathrm{mg} \mathrm{mL}^{-1}$ of each enzyme to the solution of GXXG and GXG. The temperatures chosen in the reactions were $45^{\circ} \mathrm{C}(\mathrm{XacBglA}), 25^{\circ} \mathrm{C}(\mathrm{XacBglB})$ or $35^{\circ} \mathrm{C}(\mathrm{XacBglC})$. Samples were collected at $0,30 \mathrm{~min}, 1,2,3$, and $24 \mathrm{~h}$ and reactions were stopped by the addition of methanol. A total of $5 \mu \mathrm{L}$ of the quenched reactions were added to $95 \mu \mathrm{L}$ of $5 \mu \mathrm{mol} \mathrm{L}^{-1}$ xylohexaose (used as the internal standard) in water and injected into an LTQ XL TM linear ion trap mass spectrometer (Thermo Fisher Scientific) in scan mode $(\mathrm{m} / z$ 300-1300). Samples were directly infused at a rate of $10 \mu \mathrm{L} \mathrm{min}{ }^{-1}$ into an $\operatorname{ESI}(+)$ source with a spray voltage maintained at $4.0 \mathrm{kV}$ and heated to $250{ }^{\circ} \mathrm{C}$ in the source.

The xyloglucanase activity of wild-type and mutant Xanthomonas strains were monitored in plate assays. Cultures were grown overnight in LBON medium (1\% $\mathrm{m} / v$ bacto peptone and $0.5 \% \mathrm{~m} / v$ yeast extract) at $30{ }^{\circ} \mathrm{C}$ and $200 \mathrm{rpm}$, diluted to $\mathrm{OD}_{600 \mathrm{~nm}} 0.4$, plated $(0.5 \mu \mathrm{L})$ on solid NYG medium $\left(5 \mathrm{~g} \mathrm{~L}^{-1}\right.$ peptone, $3 \mathrm{~g} \mathrm{~L}^{-1}$ yeast extract, $20 \mathrm{~g} \mathrm{~L}^{-1}$ glycerol, $15 \mathrm{~g} \mathrm{~L}^{-1}$ agar) supplemented with $0.5 \%$ of tamarind xyloglucan (Megazyme), and grown for $40 \mathrm{~h}$ at $30^{\circ} \mathrm{C}$. Activity halos were revealed with $5 \mathrm{mg} \mathrm{mL}^{-1}$ Congo red and successive washes with $1 \mathrm{~mol} \mathrm{~L}^{-1} \mathrm{NaCl}$.

Arabidopsis thaliana cultivation and XyGOs enzymatic extraction. Arabidopsis thaliana Col-0 seeds were sterilized in 70\% ethanol solution for $2 \mathrm{~min}$ in a $2 \mathrm{~mL}$ tube. The supernatant was discarded and followed by addition of $10 \%(v / v)$ sodium hypochlorite, $10 \mu \mathrm{L}$ Tween 20 (Sigma-Aldrich) solution for 5 min under agitation. Then, the material was washed five times with sterile water and stratificated in water for $48 \mathrm{~h}$ at $4{ }^{\circ} \mathrm{C}$ protected from light. Seeds were plated over Murashige and Skoog ${ }^{78}$ sterile media (Sigma-Aldrich) at half strength with $10 \%$ agar. Plates were exposed to a photosynthetic photon flux density of $200 \mu \mathrm{mol} \mathrm{m}^{-2} \mathrm{~s}^{-1}$ for $2 \mathrm{~h}$ and then grew in a Phytotron chamber (Fitotron HGC Weiss Technik) for 6 days in the dark at $21^{\circ} \mathrm{C}, 70 \%$ humidity. Seedlings (around $1 \mathrm{~cm}$ long in height) were harvested, weighted, frozen in liquid nitrogen and stored at $-80^{\circ} \mathrm{C}^{79}$.

Frozen seedlings were homogenized in a Retschmill (model MM200, Retsch) at $25 \mathrm{~Hz}$ for $1 \mathrm{~min}$. The grounded plants were washed three times by resuspending it in $1 \mathrm{~mL}$ methanol, vortexing, centrifuging at $10,000 \mathrm{~g}$ for $10 \mathrm{~min}$ and discarding the supernatant. The material was dried for $5 \mathrm{~min}$ under vacuum and washed twice with $500 \mu \mathrm{L}$ of water, discarding the supernatant ${ }^{79}$. The resulting residue was used for acetylated XyGOs extraction. A proportion of $1 \mu \mathrm{g}$ of purified XacXeg74 enzyme for every $50 \mathrm{mg}$ of starting seedlings was utilized for digesting the acetylated XyG in $200 \mu \mathrm{L}$ of $50 \mathrm{mmol} \mathrm{L}^{-1}$ ammonium formate $\mathrm{pH} 5.0$ solution for $16 \mathrm{~h}$ at $30^{\circ} \mathrm{C}, 450 \mathrm{rpm}$. Reaction was stopped by heating the mixture for $2 \mathrm{~min}$ at $95^{\circ} \mathrm{C}$. The resulting solution was centrifuged twice at $1000 \mathrm{~g}$ for $5 \mathrm{~min}$, the pellet discarded and the supernatant finally stored at $-20^{\circ} \mathrm{C}$ upon utilization.

Xyloglucan acetylesterase assays. Acetylated mono- and disaccharides were chemically prepared with excess of acetic anhydride in the presence of the catalyst pyridine ${ }^{80}$ and validated by ${ }^{1} \mathrm{H}$ nuclear magnetic resonance. Spectra analysis showed that the acetylation on both monosaccharides (mannose pentaacetate, galactose pentaacetate, fucose tetraacetate, arabinose tetraacetate and xylose tetraacetate) and disaccharides ( $\beta$-1,4-glucobiose octaacetate and saccharose octaacetate) was not selective. The ${ }^{1} \mathrm{H}$ nuclear magnetic resonance spectra were recorded on DD2 spectrometer (Agilent) from Brazilian Biosciences National Laboratory (LNBio-CNPEM, Campinas, Brazil), operating in Larmor frequency of 499.726 MHz equipped with triple resonance probe. NMR data processing was performed using VnmrJ software (4.2 Revision A). Other used acetylated sugars were purchased including $N$-acetylglucosamine, $N$-acetylneuraminic acid (SigmaAldrich), $\alpha$-glucose pentaacetate (Santa Cruz Biotechnology), $\beta$-galactose pentaacetate and $\beta$-glucose pentaacetate (Combi-Blocks). Reactions consisted of $0.01 \mathrm{mg}$ $\mathrm{mL}^{-1}$ of the enzyme XacXaeA and $5 \mathrm{mmol} \mathrm{L}^{-1}$ of each acetylated sugars incubated during $15 \mathrm{~min}$ at $20^{\circ} \mathrm{C}$ and $600 \mathrm{rpm}$ in $50 \mathrm{mmol} \mathrm{L}^{-1} \mathrm{HEPES}$ buffer $\mathrm{pH} 7.5$. 
Reactions were stopped by adding $40 \mu \mathrm{L}$ of methanol. Final products and residual substrates were monitored on a Waters Synapt HDMS system at V mode, and ESI $(+)$ with a spray voltage maintained at $3.0 \mathrm{kV}$ and heated to $130^{\circ} \mathrm{C}$ in the source using MassLynx 4.1 software. A volume of $15 \mu \mathrm{L}$ of the quenched reactions and $2 \mu \mathrm{L}$ of $1 \mathrm{mmol} \mathrm{L}^{-1}$ xylotetraose (used as the internal standard) were added to $183 \mu \mathrm{L}$ of water and injected into the mass spectrometer in scan mode $(\mathrm{m} / \mathrm{z}$ $150-900)$ with direct infusion at a flow rate of $50 \mu \mathrm{L} \mathrm{min}-181$

Esterase reactions on acetylated XyGOs extracted from A. thaliana (see section above) consisted of incubating $0.02 \mathrm{mg} \mathrm{mL}^{-1}$ of XacXaeA with the substrate (estimated concentration $5-10 \mu \mathrm{g} \mathrm{mL}^{-1}$ ) for 2 and $24 \mathrm{~h}$ at $20^{\circ} \mathrm{C}$ and $600 \mathrm{rpm}$ on $50 \mathrm{mmol} \mathrm{L}^{-1}$ HEPES buffer $\mathrm{pH}$ 7.5. $100 \mu \mathrm{L}$ of quenched reactions were desalted using Oasis HLB cartridges (Waters). HLB cartridges were first activated with methanol and equilibrated with water according to the manufacturer's protocol. The samples were applied and then washed seven times with $1 \mathrm{~mL}$ water. XyGOs were eluted with $150 \mu \mathrm{L} 25 \%(v / v)$ methanol in water. Controls, final products and residual substrates were analyzed on a LTQ XL TM linear ion trap mass spectrometer (Thermo Fisher Scientific). The samples were directly infused at a rate of $10 \mu \mathrm{L} \mathrm{min}^{-1}$ into the ESI $(+)$ source in scan mode $(\mathrm{m} / z$ 150-2000). The spray voltage maintained at $4.2 \mathrm{kV}$ and heated to $280^{\circ} \mathrm{C}$ in the source. CID-MS/MS fragmentation analysis of XyGOs were performed using different collision energies (15-35) and the isolation window was set to $1 \mathrm{Th}$. Estimated mass/charge ratio of acetylated and non-acetylated oligosaccharides were compatible to the literature $^{44,79}$ and confirmed by MS/MS fragmentation fingerprint.

Xanthomonas cultivations. For growth curve analysis, $X$. citri strains was cultured in LBON medium ( $1 \% \mathrm{~m} / v$ bacto peptone and $0.5 \% \mathrm{~m} / \mathrm{v}$ yeast extract) containing $100 \mu \mathrm{g} \mathrm{mL} \mathrm{L}^{-1}$ ampicillin at $30^{\circ} \mathrm{C}$ and $200 \mathrm{rpm}$ until mid-exponential phase. Then, the harvested cells were washed once and transferred to the modified minimal medium XVM2 $2^{52}$ (XVM2m, without sucrose and fructose, containing different sugar sources at a final concentration of $5 \mathrm{mg} \mathrm{mL}^{-1}$ ), for an initial $\mathrm{OD}_{600 \mathrm{~nm}}=0.01$. Growth was monitored for $30 \mathrm{~h}$, at $30^{\circ} \mathrm{C}$, in a SpectraMax M3 Multi-Mode Microplate Reader (Molecular Devices). Four biological replicates were used for each condition. XyGOs used in Xanthomonas growth assays were prepared by incubating $5 \mathrm{mg} \mathrm{mL}^{-1}$ tamarind xyloglucan (Megazyme) with XacXeg74 $\left(4 \mu \mathrm{g} \mathrm{mL}^{-1}\right)$ at $30^{\circ} \mathrm{C}$ for $14 \mathrm{~h}$. The reaction was stopped by heating at $80^{\circ} \mathrm{C}$ for $15 \mathrm{~min}$.

RNA sequencing and analysis. Total RNA was extracted from $15 \mathrm{~mL}$ of $X$. citri cultures grown on XVM2m + XyGOs or XVM2m + glucose medium (see the section above) at the mid-exponential phase using the TRIzol/chloroform protocol ${ }^{82}$. Samples were further treated with RNase-free DNaseI (Invitrogen) and RNaseOUT (Invitrogen) and purified with the RNeasy Mini Kit (Qiagen), according to the manufacturer's recommendations. In addition, RNA integrity was evaluated in an Agilent 2100 Bioanalyzer (Agilent Technologies) and samples were quantified in a Qubit ${ }^{\circledR}$ 2.0 Fluorometer using the RNA BR assay kit (Life Technologies). Libraries were prepared according to the manufacturer's protocol of the TruSeq Stranded Total RNA kit (Illumina Inc.). Sequencing was performed on the Illumina HiSeq 2500 platform (LNBR-CNPEM, Campinas, Brazil). RNA-seq data were deposited in the Gene Expression Omnibus database under accession number GSE159288.

RNA-seq raw reads were filtered to remove low-quality reads and adapters sequences using Trimommatic v. $0.38^{83}$ and rRNA reads were removed using SortMeRNA $2.1^{84}$ (Supplementary Table 22). High-quality reads were mapped to the Xanthomonas citri pv. citri 306 genome $\mathrm{e}^{85}$ using Bowtie2 v.2.2.5 algorithm ${ }^{86}$ and reproducibility among the biological replicates was assessed by the Principal Component Analysis and Pearson correlation methods. Differential expression analysis was carried out by pairwise comparison between X. citri grown in XVM2m containing XyGOs and XVM2m glucose medium using $\mid \log _{2}$ Fold Change $\mid \geq 1$ and a $p$ adjusted $\leq 0.05$ as thresholds using the Bioconductor DESeq2 v.1.18.1 ${ }^{87}$ package in the R v.3.4.1 platform $^{88}$.

RT-qPCR analysis. RNA-seq data were analyzed for the identification of potential reference genes. The arithmetic mean of the TPM values (transcription per million reads) of each gene was calculated in all conditions, followed by the determination of the variation coefficient and MFC (ratio between the maximum and minimum TPM value of each gene $)^{89,90}$. From the 20 potentially most stable genes, the targets XAC2293, XAC2177, XAC4047, and XAC4218 were selected for RT-qPCR experiments based on their mean TPM and $p$ values (Supplementary Table 23). The expression stability was evaluated for the potential reference genes and the $\mathrm{Cq}$ values (quantification cycles) were analyzed using three different statistical tools: BestKeeper ${ }^{91}$, NormFinder ${ }^{92}$ and RefFinder ${ }^{93}$ (Supplementary Table 24, and Supplementary Fig. 29). RT-qPCR assays were performed in an Applied Biosystems ViiA $^{m} 7$ Real-Time equipment (Life Technologies) using the Power SYBR ${ }^{\oplus}$ Green RNA-to-CT ${ }^{\text {mo }} 1$-Step Kit (Life Technologies) as detailed in Supplementary Table 25. The relative normalized expression values for each gene were calculated according to the $2^{-\Delta \Delta C t}$ method $^{94}$. Data were log-transformed and statistically compared by ANOVA and unpaired 2-tailed $t$ test using Prism 8.4.1 software (GraphPad). The correlation between gene expression data obtained in RNA-seq and RT-qPCR assays can be assessed in Supplementary Fig. 30.
Gene knockout in X. citri. Single $(\triangle \mathrm{XAC1768,} \triangle \mathrm{XAC1769,} \triangle \mathrm{XAC1770,}$ $\triangle \mathrm{XAC} 1777)$ and double ( $\triangle \mathrm{XAC1768-XAC1769)}$ gene knockout mutants were obtained by a two-step allelic exchange procedure. DNA fragments $(\sim 1.2 \mathrm{~kb})$ corresponding to regions upstream and downstream to the target genes were amplified by PCR from the X. citri genome (Supplementary Table 26). Each corresponding pair of fragments was ligated and then cloned into the pNPTS138 suicide vector ${ }^{95}$ in the corresponding restriction sites. The plasmids were introduced into $X$. citri by electroporation $(\sim 2.3 \mathrm{kV}, \sim 5 \mathrm{~ms})$, and sucrose-sensitive and kanamycin-resistant colonies were selected (LBON-agar, $100 \mu \mathrm{g} \mathrm{mL}^{-1}$ ampicillin, $100 \mu \mathrm{g} \mathrm{mL}^{-1}$ kanamycin with and without $5 \%$ sucrose, respectively). This step selected colonies that suffered the first homologous recombination event, when the plasmid is inserted into the bacterial genome. These colonies were grown in LBON, $100 \mu \mathrm{gL}^{-1}$ ampicillin without selection to allow the occurrence of the second recombinant event, when the plasmid is excised from the genome. The cultures were plated, and individual colonies were selected for simultaneous sucrose resistance and kanamycin sensitivity. Deletions were confirmed by PCR and DNA-sequencing (Supplementary Table 27).

Virulence assays. Plants of sweet orange (Citrus sinensis 'Natal') were infiltrated by the pinprick method ${ }^{96}$ with water suspensions of $X$. citri at $\mathrm{OD}_{600}$ of 0.1 previously grown in LBON agar plates, supplemented with ampicillin $\left(100 \mu \mathrm{g} \mathrm{mL} \mathrm{L}^{-1}\right)$, for $48 \mathrm{~h}$ at $28^{\circ} \mathrm{C}$. The assays were performed with three independent biological samples, each composed of 16 technical replicates. Plants were maintained under greenhouse conditions and monitored daily for the appearance of canker symptoms. Quantitative analyses of canker lesions were performed using ImageJ v. 1.53b.

Reporting summary. Further information on research design is available in the Nature Research Reporting Summary linked to this article.

\section{Data availability}

Atomic coordinates and structure factors have been deposited in the Protein Data Bank (PDB) with accession codes 7KN8 (XccXeg74 complexed with XG oligosaccharide), 7KMM (native XacXaeA), 7KMN (native XacGalD), 7KMO (XacGalD complexed with galactose), 7KMP (native XacXyl31), 7KNC (XacXyl31 complexed with xylose) and 7KMQ (native XacAfc95). RNA-seq data were deposited in the Gene Expression Omnibus database under accession number GSE159288. Additional data that support the findings of this study are available from the corresponding authors on reasonable request. Source data are provided with this paper.

Received: 30 November 2020; Accepted: 7 June 2021; Published online: 30 June 2021

\section{References}

1. Eckardt, N. A. Role of xyloglucan in primary cell walls. Plant Cell 20, 1421-1422 (2008).

2. O'Neill, M. A. \& York, W. S. The Composition and Structure of Plant Primary Cell Walls. in Annual Plant Reviews, 1-54 (John Wiley \& Sons, Ltd, Circhester, 2018).

3. Waldron, K. W. \& Faulds, C. B. Cell wall polysaccharides: composition and structure. in Comprehensive Glycoscience Ch. 5 1, 181-201 (Elsevier, Amsterdam, 2007).

4. Nishinari, K., Takemasa, M., Zhang, H. \& Takahashi, R. Storage plant polysaccharides: xyloglucans, galactomannans, glucomannans. in Comprehensive Glycoscience 613-652 (Elsevier, Amsterdam, 2007).

5. Larsbrink, J. et al. A complex gene locus enables xyloglucan utilization in the model saprophyte Cellvibrio japonicus. Mol. Microbiol. 94, 418-433 (2014).

6. Larsbrink, J. et al. A discrete genetic locus confers xyloglucan metabolism in select human gut Bacteroidetes. Nature 506, 498-502 (2014).

7. Ryan, R. P. et al. Pathogenomics of Xanthomonas: understanding bacteriumplant interactions. Nat. Rev. Microbiol. 9, 344-355 (2011).

8. Timilsina, S. et al. Xanthomonas diversity, virulence and plant-pathogen interactions. Nat. Rev. Microbiol. 18, 415-427 (2020).

9. Brunings, A. M. \& Gabriel, D. W. Xanthomonas citri: Breaking the surface. Mol. Plant Pathol. 4, 141-157 (2003).

10. Kay, S. \& Bonas, U. How Xanthomonas type III effectors manipulate the host plant. Curr. Opin. Microbiol. 12, 37-43 (2009).

11. Terrapon, N. et al. PULDB: The expanded database of Polysaccharide Utilization Loci. Nucleic Acids Res. 46, D677-D683 (2018).

12. Déjean, G., Tauzin, A. S., Bennett, S. W., Creagh, A. L. \& Brumer, H. Adaptation of syntenic xyloglucan utilization loci of human gut Bacteroidetes to polysaccharide side chain diversity. Appl. Environ. Microbiol. 85, e01491-19 (2019)

13. de Souza, A. P., Leite, D. C. C., Pattathil, S., Hahn, M. G. \& Buckeridge, M. S Composition and structure of sugarcane cell wall polysaccharides: 
implications for second-generation bioethanol production. Bioenergy Res. 6 , 564-579 (2013).

14. Vogel, J. Unique aspects of the grass cell wall. Curr. Opin. Plant Biol. 11, 301-307 (2008).

15. Brennan, M., Fakharuzi, D. \& Harris, P. J. Occurrence of fucosylated and nonfucosylated xyloglucans in the cell walls of monocotyledons: an immunofluorescence study. Plant Physiol. Biochem. 139, 428-434 (2019).

16. Brennan, M. \& Harris, P. J. Distribution of fucosylated xyloglucans among the walls of different cell types in monocotyledons determined by immunofluorescence microscopy. Mol. Plant 4, 144-156 (2011).

17. An, S. Q. et al. Mechanistic insights into host adaptation, virulence and epidemiology of the phytopathogen. Xanthomonas. FEMS Microbiol. Rev. 44, $1-32$ (2019).

18. Matsuzawa, T. \& Yaoi, K. GH74 xyloglucanases: structures and modes of activity. Trends Glycosci. Glycotechnol. 28, E63-E70 (2016).

19. Arnal, G. et al. Substrate specificity, regiospecificity, and processivity in glycoside hydrolase family 74. J. Biol. Chem. 294, 13233-13247 (2019).

20. Lombard, V., Golaconda Ramulu, H., Drula, E., Coutinho, P. M. \& Henrissat, B. The carbohydrate-active enzymes database (CAZy) in 2013. Nucleic Acids Res. 42, D490-D495 (2014).

21. Zhong, R., Cui, D. \& Ye, Z. H. Xyloglucan O-acetyltransferases from Arabidopsis thaliana and Populus trichocarpa catalyze acetylation of fucosylated galactose residues on xyloglucan side chains. Planta 248, 1159-1171 (2018).

22. Nardini, M. \& Dijkstra, B. W. $\alpha / \beta$ hydrolase fold enzymes: the family keeps growing. Curr. Opin. Struct. Biol. 9, 732-737 (1999).

23. Montanier, C. et al. The active site of a carbohydrate esterase displays divergent catalytic and noncatalytic binding functions. PLoS Biol. 7, 0687-0697 (2009).

24. Correia, M. A. S. et al. Crystal structure of a cellulosomal family 3 carbohydrate esterase from Clostridium thermocellum provides insights into the mechanism of substrate secognition. J. Mol. Biol. 379, 64-72 (2008).

25. Bitto, E. et al. The structure at $1.6 \AA$ resolution of the protein product of the At4g34215 gene from Arabidopsis thaliana. Acta Crystallogr. Sect. D. Biol. Crystallogr. 61, 1655-1661 (2005).

26. Mølgaard, A., Kauppinen, S. \& Larsen, S. Rhamnogalacturonan acetylesterase elucidates the structure and function of a new family of hydrolases. Structure 8, 373-383 (2000).

27. Michalak, L. et al. A pair of esterases from a commensal gut bacterium remove acetylations from all positions on complex $\beta$-mannans. Proc. Natl Acad. Sci. USA 117, 7122-7130 (2020).

28. Sun, Y. et al. Molecular basis of the general base catalysis of an $\alpha / \beta$ - hydrolase catalytic triad. J. Biol. Chem. 289, 15867-15879 (2014).

29. Rauwerdink, A. \& Kazlauskas, R. J. How the same core catalytic machinery catalyzes 17 different reactions: the serine-histidine-aspartate catalytic triad of $\alpha / \beta$-hydrolase fold enzymes. ACS Catal. 5, 6153-6176 (2015).

30. Akoh, C. C., Lee, G. C., Liaw, Y. C., Huang, T. H. \& Shaw, J. F. GDSL family of serine esterases/lipases. Prog. Lipid Res. 43, 534-552 (2004).

31. Ménard, R. \& Storer, A. C. Oxyanion hole interactions in serine and cysteine proteases. Biol. Chem. Hoppe. Seyler. 373, 393-400 (1992).

32. Rogowski, A. et al. Glycan complexity dictates microbial resource allocation in the large intestine. Nat. Commun. 6, 7481 (2015).

33. Nagae, M. et al. Structural basis of the catalytic reaction mechanism of novel 1,2- $\alpha$-L-fucosidase from Bifidobacterium bifidum. J. Biol. Chem. 282, 18497-18509 (2007).

34. Sela, D. A. et al. Bifidobacterium longum subsp. infantis ATCC 15697 afucosidases are active on fucosylated human milk oligosaccharides. Appl. Environ. Microbiol. 78, 795-803 (2012).

35. Yang, T. C., Hu, R. M., Weng, S. F. \& Tseng, Y. H. Identification of a hypothetical protein of plant pathogenic Xanthomonas campestris as a novel $\beta$-galactosidase. J. Mol. Microbiol. Biotechnol. 13, 172-180 (2007).

36. Cao, H., Walton, J. D., Brumm, P. \& Phillips, G. N. Crystal structure of $\alpha-$ xylosidase from Aspergillus niger in complex with a hydrolyzed xyloglucan product and new insights in accurately predicting substrate specificities of GH31 family glycosidases. ACS Sustain. Chem. Eng. 8, 2540-2547 (2020).

37. Larsbrink, J. et al. Structural and enzymatic characterization of a glycoside hydrolase family $31 \alpha$-xylosidase from Cellvibrio japonicus involved in xyloglucan saccharification. Biochem. J. 436, 567-580 (2011).

38. Rigden, D. J., Mello, L. V. \& Galperin, M. Y. The PA14 domain, a conserved all- $\beta$ domain in bacterial toxins, enzymes, adhesins and signaling molecules. Trends Biochem. Sci. 29, 335-339 (2004).

39. Yoshida, E. et al. Role of a PA14 domain in determining substrate specificity of a glycoside hydrolase family $3 \beta$-glucosidase from Kluyveromyces marxianus. Biochem. J. 431, 39-49 (2010).

40. Hemsworth, G. R. et al. Structural dissection of a complex Bacteroides ovatus gene locus conferring xyloglucan metabolism in the human gut. Open Biol. 6, 160142 (2016).
41. Silipo, A. et al. NMR spectroscopic analysis reveals extensive binding interactions of complex xyloglucan oligosaccharides with the Cellvibrio japonicus glycoside hydrolase family 31 a-xylosidase. Chem. Eur. J. 18, 13395-13404 (2012).

42. Nelson, C. E. et al. Comprehensive functional characterization of the glycoside hydrolase family 3 enzymes from Cellvibrio japonicus reveals unique metabolic roles in biomass saccharification. Environ. Microbiol. 19, 5025-5039 (2017).

43. Mewis, K., Lenfant, N., Lombard, V. \& Henrissat, B. Dividing the large glycoside hydrolase family 43 into subfamilies: A motivation for detailed enzyme characterization. Appl. Environ. Microbiol. 82, 1686-1692 (2016).

44. Hsieh, Y. S. Y. \& Harris, P. J. Xyloglucans of monocotyledons have diverse structures. Mol. Plant 2, 943-965 (2009).

45. Hoffman, M. et al. Structural analysis of xyloglucans in the primary cell walls of plants in the subclass. Asteridae. Carbohydr. Res. 340, 1826-1840 (2005).

46. Facincani, A. P. et al. Comparative proteomic analysis reveals that T3SS, Tfp and xanthan gum are key factors in initial stages of Citrus sinensis infection by Xanthomonas citri subsp. citri. Funct. Integr. Genom. 14, 205-217 (2014).

47. Guo, Y., Figueiredo, F., Jones, J. \& Wang, N. HrpG and HrpX play global roles in coordinating different virulence traits of Xanthomonas axonopodis pv. citri. Mol. Plant-Microbe Interact. 24, 649-661 (2011).

48. de Melo, R. R. et al. Identification of a cold-adapted and metal-stimulated $\beta$ 1,4-glucanase with potential use in the extraction of bioactive compounds from plants. Int. J. Biol. Macromol. 166, 190-199 (2021).

49. Aspeborg, H., Coutinho, P. M., Wang, Y., Brumer, H. \& Henrissat, B. Evolution, substrate specificity and subfamily classification of glycoside hydrolase family 5 (GH5). BMC Evol. Biol. 12, 186 (2012).

50. Hudson, K. L. et al. Carbohydrate-aromatic interactions in proteins. J. Am. Chem. Soc. 137, 15152-15160 (2015).

51. Arlat, M., Gough, C. L., Barber, C. E., Boucher, C. \& Daniels, M. J. Xanthomonas campestris contains a cluster of $h r p$ genes related to the larger $h r p$ cluster of Pseudomonas solanacearum. Mol. Plant. Microbe Interact. 4, 593-601 (1991).

52. Wengelnik, K., Marie, C., Russel, M. \& Bonas, U. Expression and localization of HrpA1, a protein of Xanthomonas campestris pv. vesicatoria essential for pathogenicity and induction of the hypersensitive reaction. J. Bacteriol. 178 1061-1069 (1996)

53. Tsuge, S. et al. Expression of Xanthomonas oryzae pv. oryzae hrp genes in XOM2, a novel synthetic medium. J. Gen. Plant Pathol. 68, 363-371 (2002).

54. Ikawa, Y. \& Tsuge, S. The quantitative regulation of the $h r p$ regulator HrpX is involved in sugar-source-dependent $h r p$ gene expression in Xanthomonas oryzae pv. oryzae. FEMS Microbiol. Lett. 363, fnw071 (2016).

55. Ikawa, Y., Ohnishi, S., Shoji, A., Furutani, A. \& Tsuge, S. Concomitant regulation by a LacI-Type transcriptional repressor XylR on genes involved in xylan and xylose metabolism and the type III secretion system in rice pathogen Xanthomonas oryzae pv. oryzae. Mol. Plant-Microbe Interact. 31, 605-613 (2018).

56. Rashid, M. M., Ikawa, Y. \& Tsuge, S. GamR, the LysR-type galactose metabolism regulator, regulates $h r p$ gene expression via transcriptional activation of two key hrp regulators, HrpG and HrpX, in Xanthomonas oryzae pv. oryzae. Appl. Environ. Microbiol. 82, 3947-3958 (2016).

57. Déjean, G. et al. The xylan utilization system of the plant pathogen Xanthomonas campestris pv. campestris controls epiphytic life and reveals common features with oligotrophic bacteria and animal gut symbionts. $N$. Phytol. 198, 899-915 (2013).

58. Santos, C. R. et al. Molecular mechanisms associated with xylan degradation by Xanthomonas plant pathogens. J. Biol. Chem. 289, 32186-32200 (2014)

59. Tayi, L., Maku, R. V., Patel, H. K. \& Sonti, R. V. Identification of pectin degrading enzymes secreted by Xanthomonas oryzae pv. oryzae and determination of their role in virulence on rice. PLOS ONE 11, 1-15 (2016).

60. Yu, G., Wang, L. G., Han, Y. \& He, Q. Y. ClusterProfiler: an R package for comparing biological themes among gene clusters. Omi. A J. Integr. Biol. 16, 284-287 (2012).

61. Na, S. I. et al. UBCG: Up-to-date bacterial core gene set and pipeline for phylogenomic tree reconstruction. J. Microbiol. 56, 281-285 (2018).

62. de Sousa, A. S. et al. A rationally identified marine GH1 $\beta$-glucosidase has distinguishing functional features for simultaneous saccharification and fermentation. Biofuels. Bioprod. Bioref. 14, 1163-1179 (2020).

63. Franke, D. et al. ATSAS 2.8: A comprehensive data analysis suite for smallangle scattering from macromolecular solutions. J. Appl. Crystallogr. 50, 1212-1225 (2017)

64. Piiadov, V., Ares de Araújo, E., Oliveira Neto, M., Craievich, A. F. \& Polikarpov, I. SAXSMoW 2.0: online calculator of the molecular weight of proteins in dilute solution from experimental SAXS data measured on a relative scale. Protein Sci. 28, 454-463 (2019).

65. Krissinel, E. \& Henrick, K. 'Protein interfaces, surfaces and assemblies' service PISA at the European Bioinformatics Institute. (http://www.ebi.ac.uk/pdbe/ prot_int/pistart.html), inference of macromolecular assemblies from crystalline state. J. Mol. Biol. 372, 774-797 (2007).

66. Kabsch, W. X. D. S. Acta, Crystallogr \& Sect, D. Biol. Crystallogr. 66, 125-132 (2010). 
67. McCoy, A. J. et al. Phaser crystallographic software. J. Appl. Crystallogr. 40, 658-674 (2007).

68. Martinez-Fleites, C. et al. Crystal structures of Clostridium thermocellum xyloglucanase, XGH74A, reveal the structural basis for xyloglucan recognition and degradation. J. Biol. Chem. 281, 24922-24933 (2006).

69. Sheldrick, G. M. Experimental phasing with SHELXC/D/E: Combining chain tracing with density modification. Acta Crystallogr. Sect. D. Biol. Crystallogr. 66, 479-485 (2010).

70. Liebschner, D. et al. Macromolecular structure determination using X-rays, neutrons and electrons: recent developments in Phenix. Acta Crystallogr. Sect. D. Struct. Biol. 75, 861-877 (2019).

71. Emsley, P., Lohkamp, B., Scott, W. G. \& Cowtan, K. Features and development of Coot. Acta Crystallogr. Sect. D. Biol. Crystallogr. 66, 486-501 (2010).

72. Davis, I. W. et al. MolProbity: all-atom contacts and structure validation for proteins and nucleic acids. Nucleic Acids Res 35, W375-W383 (2007).

73. Zheng, H. et al. Validation of metal-binding sites in macromolecular structures with the CheckMyMetal web server. Nat. Protoc. 9, 156-170 (2014).

74. Zheng, H. et al. CheckMyMetal: a macromolecular metal-binding validation tool. Acta Crystallogr. Sect. D. Struct. Biol. 73, 223-233 (2017).

75. Handing, K. B. et al. Characterizing metal-binding sites in proteins with X-ray crystallography. Nat. Protoc. 13, 1062-1090 (2018).

76. Agirre, J. et al. Privateer: software for the conformational validation of carbohydrate structures. Nat. Struct. Mol. Biol. 22, 833-834 (2015).

77. Buckeridge, M. S., Rocha, D. C., Reid, J. S. G. \& Dietrich, S. M. C. Xyloglucan structure and post-germinative metabolism in seeds of Copaifera langsdorfii from savanna and forest populations. Physiol. Plant. 86, 145-151 (1992).

78. Murashige, T. \& Skoog, F. A revised medium for rapid growth and bio assays with tobacco tissue cultures. Physiol. Plant. 15, 473-497 (1962).

79. Lerouxel, O. et al. Rapid structural phenotyping of plant cell wall mutants by enzymatic oligosaccharide fingerprinting. Plant Physiol. 130, 1754-1763 (2002).

80. Höfle, G., Steglich, W. \& Vorbrüggen, H. 4-dialkylaminopyridines as highly active acylation catalysts. [new synthetic method (25)]. Angew. Chem. Int. Ed. 17, 569-583 (1978)

81. Dos Santos, C. R. et al. The mechanism by which a distinguishing arabinofuranosidase can cope with internal di-substitutions in arabinoxylans. Biotechnol. Biofuels 11, 223 (2018).

82. Chomczynski, P. \& Mackey, K. Modification of the TRI Reagent(TM) procedure for isolation of RNA from polysaccharide- and proteoglycan-rich sources. Biotechniques 19, 942-945 (1995).

83. Bolger, A. M., Lohse, M. \& Usadel, B. Trimmomatic: a flexible trimmer for Illumina sequence data. Bioinformatics 30, 2114-2120 (2014).

84. Kopylova, E., Noé, L. \& Touzet, H. SortMeRNA: fast and accurate filtering of ribosomal RNAs in metatranscriptomic data. Bioinformatics 28, 3211-3217 (2012).

85. Da Silva, A. C. R. et al. Comparison of the genomes of two Xanthomonas pathogens with differing host specificities. Nature 417, 459-463 (2002).

86. Langmead, B. \& Salzberg, S. L. Fast gapped-read alignment with Bowtie 2. Nat. Methods 9, 357-359 (2012).

87. Love, M. I., Huber, W. \& Anders, S. Moderated estimation of fold change and dispersion for RNA-seq data with DESeq2. Genome Biol. 15, 55 (2014).

88. Ihaka, R. \& Gentleman, R. R: a language for data analysis and graphics. J. Comput. Graph. Stat. 5, 299 (1996).

89. Carmona, R. et al. Automated identification of reference genes based on RNAseq data. Biomed. Eng. Online 16, 65 (2017).

90. Hoang, V. L. T. et al. RNA-seq reveals more consistent reference genes for gene expression studies in human non-melanoma skin cancers. PeerJ 2017, e3631 (2017).

91. Pfaffl, M. W., Tichopad, A., Prgomet, C. \& Neuvians, T. P. Determination of stable housekeeping genes, differentially regulated target genes and sample integrity: BestKeeper-Excel-based tool using pair-wise correlations. Biotechnol. Lett. 26, 509-515 (2004).

92. Andersen, C. L., Jensen, J. L. \& Ørntoft, T. F. Normalization of real-time quantitative reverse transcription-PCR data: a model-based variance estimation approach to identify genes suited for normalization, applied to bladder and colon cancer data sets. Cancer Res. 64, 5245-5250 (2004).

93. Xie, F., Xiao, P., Chen, D., Xu, L. \& Zhang, B. miRDeepFinder: a miRNA analysis tool for deep sequencing of plant small RNAs. Plant Mol. Biol. 80 75-84 (2012)

94. Pfaffl, M. W. A new mathematical model for relative quantification in realtime RT-PCR. Nucleic Acids Res. 29, 45e-45e (2001).

95. Ried, J. L. \& Collmer, A. An nptI-sacB-sacR cartridge for constructing directed, unmarked mutations in gram-negative bacteria by marker exchangeeviction mutagenesis. Gene 57, 239-246 (1987)

96. Soprano, A. S. et al. Crystal structure and regulation of the Citrus Pol III repressor MAF1 by auxin and phosphorylation. Structure 25, 1360-1370.e4 (2017)

\section{Acknowledgements}

We are grateful to Dr. Felipe Rafael Torres for the discussions on the RT-qPCR analysis, to Dr. Nicolas Terrapon and the CAZy database for analyzing and establishing the novel CE20 family, to Dr. Juliana Velasco de Castro Oliveira for the support in the cultivation of A. thaliana and all the staff of the several facilities listed below for their technical support We thank the Brazilian Synchrotron Light Laboratory (LNLS-CNPEM/MCTI) for the provision of time on the SAXS1 and MX2 beamlines, the Stanford Synchrotron Radiation National Accelerator Laboratory (SSRL-SLAC) for the provision of time on the BL9-2 beamline, the Brazilian Biosciences National Laboratory (LNBio-CNPEM/MCTI) for access to the crystallization (Robolab) facility, the Nuclear Magnetic Resonance (NMR) facility and the Chemistry and Natural Products Laboratory, and the Brazilian Biorenewables National Laboratory (LNBR-CNPEM/MCTI) for the use of the Next Generation Sequencing (NGS) and Characterization of Macromolecules (MAC) open access facilities. Use of the Stanford Synchrotron Radiation Lightsource, SLAC National Accelerator Laboratory, is supported by the U.S. Department of Energy, Office of Science, Office of Basic Energy Sciences under Contract No. DE-AC02-76SF00515. The SSRL Structural Molecular Biology Program is supported by the DOE Office of Biological and Environmental Research, and by the National Institutes of Health, National Institute of General Medical Sciences (P41GM103393). The contents of this publication are solely the responsibility of the authors and do not necessarily represent the official views of NIGMS or NIH. This research was funded by São Paulo Research Foundation (FAPESP, 2015/ 26982-0 to M.T.M. and 2015/13684-0 to I.P.) and by Conselho Nacional de Desenvolvimento Científico e Tecnológico (CNPq, 303988-2015-5 to I.P., and 408600/2018-7 to G.F.P.). P.S.V., R.R.M., M.A.B.M., and A.G. received post-doctoral FAPESP fellowships (2016/06509-0, 2017/14253-9, 2016/19995-0 and 2019/13936-0 respectively), I.M.B. and J. A.D. received FAPESP PhD fellowships (2017/00203-0 and 2018/03724-3 respectively), and E.A.A. received CNPq PhD fellowship (158752/2015-5).

\section{Author contributions}

P.S.V., E.A.A., E.A.L., M.R.F., R.R.M., M.A.B.M., J.B.L.C., J.A.D. performed enzyme assays. P.S.V. and L.M.Z. performed biophysical analyses. P.S.V., A.G. and M.S.B. performed carbohydrate extraction. P.S.V., E.A.A., M.A.B.M., I.P. and M.T.M. performed crystallographic studies. S.A.R. synthesized and analyzed the acetylated mono- and disaccharides. A.R.L., I.M.B., C.E.B. and P.O.G. performed gene knockout and in vivo experiments. I.M.B., D.A.A.P., G.F.P. and P.O.G. performed RNA-seq analyses. G.F.P. performed phylogenetic analyses. T.B.L., R.A.S.P., M.S.B. and F.C.G. performed and analyzed mass spectrometry experiments. I.P., P.O.G. and M.T.M. coordinated the work, analyzed the results and wrote the manuscript. P.S.V. and I.M.B. also contributed to the writing of the manuscript.

\section{Competing interests}

The authors declare no competing interests.

\section{Additional information}

Supplementary information The online version contains supplementary material available at https://doi.org/10.1038/s41467-021-24277-4.

Correspondence and requests for materials should be addressed to P.O.G. or M.T.M.

Peer review information Nature Communications thanks Ya-Wen He and the other, anonymous, reviewers for their contribution to the peer review of this work. Peer reviewer reports are available.

Reprints and permission information is available at http://www.nature.com/reprints

Publisher's note Springer Nature remains neutral with regard to jurisdictional claims in published maps and institutional affiliations.

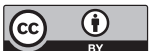

Open Access This article is licensed under a Creative Commons Attribution 4.0 International License, which permits use, sharing, adaptation, distribution and reproduction in any medium or format, as long as you give appropriate credit to the original author(s) and the source, provide a link to the Creative Commons license, and indicate if changes were made. The images or other third party material in this article are included in the article's Creative Commons license, unless indicated otherwise in a credit line to the material. If material is not included in the article's Creative Commons license and your intended use is not permitted by statutory regulation or exceeds the permitted use, you will need to obtain permission directly from the copyright holder. To view a copy of this license, visit http://creativecommons.org/ licenses/by/4.0/

(C) The Author(s) 2021 